\title{
Scattering of solitons in binary Bose-Einstein condensates with spin-orbit and Rabi couplings
}

\author{
Rafael M. P. Teixeira and Wesley B. Cardoso* \\ Instituto de Física, Universidade Federal de Goiás, 74.690-900, Goiânia, Goiás, Brazil
}

\begin{abstract}
In this paper we study the scattering of solitons in a binary Bose-Einstein Condensate (BEC) including SO- and Rabi-couplings. To this end, we derive a reduced ODE model in view to provide a variational description of the collisional dynamics. Also, we assume negative intra- and inter-component interaction strengths, such that one obtains localized solutions even in absence of external potentials. By performing extensive numerical simulations of this model we observe that, for specific conditions, the final propagation velocity of the scattered solitons could be highly sensitive to small changes in the initial conditions, being a possible signature of chaos. Additionally, there are infinitely many intervals of regularity emerging from the obtained chaotic-like regions and forming a fractal-like structure of reflection/transmission windows. Finally, we investigate how the value of the spin-orbit coupling strength changes the critical velocities, which are minimum/maximum values for the occurrence of solitons bound-states, as well as the fractal-like structure.
\end{abstract}

\section{INTRODUCTION}

Spin-orbit (SO) coupling was recently engineered in a neutral atomic Bose-Einstein condensate (BECs) by dressing two atomic spin states (hyperfine states $\left|F=1, m_{F}= \pm 1\right\rangle$ of a spin- ${ }^{87} \mathrm{Rb} \mathrm{BEC}$ ) with a pair of laser beams [1]. This new scenario has motivated further studies on vector solitons and other nonlinear waves, such as, self-trapped states [2], vortices [3-7], Skyrmions [8], Dirac monopoles [9], dark solitons [10, 11], bright solitons [12], gap solitons [13-15], exotic complexes [16], etc. Furthermore, many studies in BECs with SO coupling have shown interesting effects like the chiral confinement in quasirelativistic BECs [2], existence of a 'stripe phase' $[17,18]$, tunneling dynamics [19-21], the partial wave scattering [22], the phenomenon of Zitterbewegung [23-25], the tunability of the SO coupling strength [26], traveling Majorana solitons [27], steadily moving solitons in a helicoidal gauge potential [28], negative-mass hydrodynamics [29], etc.

Analytical developments for search localized solutions in BECs with SO coupling was recently reported in quasi-one- [11-16, 30-41] and quasi-two-dimensional [7, 15, 34, 42-46] systems. Specifically, in Ref. [30] was derived an effective 1D coupled nonpolynomial Schrödinger equations from the system of 3D GrossPitaevskii equations. Next, this study was extended to quasi-two-dimensional BECs with SO and Rabi couplings [42]. Detailed studies of stationary and moving bright solitons in BECs with SO and Rabi couplings was presented in Refs. [31, 38-41, 47] and in Refs. [35, 43] including also interatomic magnetic dipole-dipole interactions. In Ref. [32] was reported the existence of even, odd, and asymmetric nonlinear modes in the effectively 1D self-repulsive binary BEC with the SO and Zeeman splitting, confined by the axial $\mathrm{HO}$ potential.

*wesleybcardoso@gmail.com
The emergence of a number of nontrivial soliton properties due to a localized SO coupling was presented in Ref. [33]. In Ref. [34] was studied discrete and continuum composite solitons in BECs with the Rashba SO coupling loaded into a deep 1D or 2D optical-lattice potential. The spontaneous symmetry breaking in a SOcoupled $f=2$ spinor condensate was reported in [36]. In Ref. [37] was numerically investigated the ground state properties and dynamical generation of dark solitons in SO-coupled BECs. Recently, was reported in Ref. [46] the possibility to stabilize excited states of semivortex and mixed-mode solitons (originally unstable) in a setting based on repulsive dipole-dipole interactions induced by a polarizing field, oriented perpendicular to the plane in which the dipolar BEC is trapped. In addition, it has also been predicted that 2D and 3D solitons can be stabilized in spinor (two-component) BECs with the help of Rashba-type SO coupling [7, 34, 41, 43, 4852].

In a more complex scenario, collisions of solitary waves can show nontrivial structures since, due to the nonintegrability of the system, the collision outcome can depend on the initial conditions, presenting in some cases a fractal pattern [53-62]. Fractal structures in collisions of solitons are also reported in systems described by other models, such as, in the $\phi^{4}$ model $[63,64]$, the sine-Gordon model [65-69], etc. However, there are still few works dedicated to exploring collisions of localized structures in BECs with SO coupling [7, 28, 34, 44, 50]. Indeed, in Ref. [28] was reported the existence and stability of families of steadily moving solitons in a helicoidal gauge potential, where in the absence of Zeeman splitting, such solitons interact elastically similarly to solitons in integrable systems. Also, in Ref. [7] was verified that in two-dimensional SO-coupled self-attractive BECs in free space, collisions between two moving solitons lead to their merger into a single one. The scattering process due to the collisions of solitons was used in Ref. [34] in view to verify the stability of $1 \mathrm{D}$ and 2D solitons. In Ref. [44] it was studied the mobility and col- 
lision of gap-solitons in dipolar BECs with SO coupling, revealing negative and positive effective masses of the isotropic and anisotropic solitons, respectively. In addition, in Ref. [50] it was presented the study of the formation and dynamics of 2D vortex-bright solitons in a three-component $\mathrm{SO}$ coupled spin 1 spinor condensate, revealing that in the collision of two moving vortexbright solitons at small velocities, one finds that the inphase solitons either collapse or merge into a single entity, whereas out-of-phase solitons repel and avoid each other without ever having an overlapping profile. Here, we investigate the influence of the SO coupling on the collisional dynamics of solitons in BECs. To this end, we employ a reduced ordinary differential equations (ODE) model based on a variational approach, which allow us to analytically investigate the formation of fractal-like patterns and the properties of the scattered solitons.

The rest of the paper is organized as follows. In Sec. II, we describe the effective mean-field coupled GrossPitaevskii (GP) equations with SOC used to study the collisional dynamics of solitons. By means of a variational approach, we obtain a reduced ODE model in Sec. III. In Sec. IV we analyze the width oscillations in the $|\xi| \gg 1$ regime and the initial conditions to be used in the numerical simulations presented in Sec. V. Finally, in Sec. VI, we give a summary of our findings.

\section{THEORETICAL MODEL}

We start by considering a BEC confined in a quasione-dimensional parabolic trap (with frequencies $\omega_{x} \ll$ $\left.\omega_{\perp}\right)$, described by an effective 1D-GP equation system with SO and Rabi couplings, which is written in a scaled form as [12] (length in units of $a_{\perp} \equiv \sqrt{\hbar / m \omega_{\perp}}$, time in units of $\omega_{\perp}^{-1}$, and energy in units of $\hbar \omega_{\perp}$ )

$$
\begin{aligned}
i \partial_{t} A_{k}= & {\left[-\frac{1}{2} \partial_{x}^{2}+i(-1)^{k-1} \gamma \partial_{x}+V(x)\right.} \\
& \left.+g_{k}\left|A_{k}\right|^{2}+g_{12}\left|A_{3-k}\right|^{2}\right] A_{k}+\Gamma A_{3-k},
\end{aligned}
$$

where $A_{k}(k=1,2)$ are wave functions related to the two pseudospin components of the BEC. The strengths of the intra- and interspecies interactions are $g_{k} \equiv 2 a_{k} / a_{\perp}$ and $g_{12} \equiv 2 a_{12} / a_{\perp}$, with $a_{k}$ and $a_{12}$ being the respective swave scattering lengths. The strengths of the $\mathrm{SO}$ and Rabi couplings are $\gamma \equiv k_{L} a_{\perp}$ and $\Gamma \equiv \Omega /\left(2 \omega_{\perp}\right)$, respectively, where $k_{L}$ is the wave number of the Raman lasers that couple the two atomic hyperfine states in the $x$ direction [70], and $\Omega$ is the frequency of the Raman coupling, responsible for the Rabi mixing between the states.

In the following, we will assume a null interspecies interactions $g_{12}=0$ (which can be properly adjusted by means of the Feshbach resonance [71]), i.e., we consider cases where the interspecies interaction is provided only by the Rabi term. Also, in a complete attractive binary
BEC (negative $g_{1}=g_{2}=g$ and $\Gamma$ ) one can obtain localized solutions even in absence of axial confinement, because in specific conditions the self-trapping of the cigar-shaped cloud prevents spreading. In this sense, in our model we consider $V(x)=0$. In order to investigate the details of this physical process, specifically in the collisional dynamics of two solitons, in the next section we derive a reduced ODE model that aims to provide an effective description of the collision dynamics.

\section{THE REDUCED ODE MODEL}

For convenience, we reset the indexes for the components using the rule $k \rightarrow \operatorname{sgn}\left[(-1)^{k}\right](k=1,2)$. Then, we assume an approximated solution in a full functional form for symmetric bright solitons, which can be written in the form

$$
A_{ \pm}=\eta \operatorname{sech}\left(\frac{x \pm p}{w}\right) \mathrm{e}^{i\left[ \pm v(x \pm p)+\frac{b}{2 w}(x \pm p)^{2}+\sigma\right]},
$$

with the variational parameters within $A_{ \pm}$being timedependent functions, namely: amplitude $(\eta)$, velocity $(v)$, width $(w)$, peak position $(p)$, chirp $(b)$, and global phase $(\sigma)$. The exponent comes from the Galilean invariance of Eq. (1), excepting the quadratic term in $x$, which gives a parabolic phase offset to the waves that promotes width oscillations. The parameter $\sigma$ develops an important role in the model, because it is responsible for the global phase invariance of the system. Note that the momentum conservation arises naturally from the ansatz, because the total momentum of the symmetric solitons is always zero.

The Lagrangian density corresponding to Eq. (1) can be written as $\mathscr{L}=\mathcal{L}_{+}+\mathcal{L}_{-}$, in which

$$
\begin{aligned}
\mathcal{L}_{ \pm}= & \Im\left(A_{ \pm}^{*} \partial_{t} A_{ \pm}\right) \pm \gamma \Im\left(A_{ \pm}^{*} \partial_{x} A_{ \pm}\right) \\
& +\frac{1}{2}\left|\partial_{x} A_{ \pm}\right|^{2}+\frac{g_{ \pm}}{2}\left|A_{ \pm}\right|^{4}+\Gamma \Re\left(A_{ \pm}^{*} A_{\mp}\right),
\end{aligned}
$$

where $\Im(\xi)$ and $\Re(\xi)$ denote the imaginary and real parts of the complex argument $\xi$, respectively.

The variational approach yields a reduced ODE model that is calculated by substituting the ansatz (2) into the effective Lagrangian density (3), and then integrating over the whole $x$-axis. The resulting Lagrangian is given in terms of the variational parameters and their temporal derivatives, as follows

$$
\begin{aligned}
L & =4 \eta^{2} w(v \dot{p}+\dot{\sigma})+\frac{\pi^{2} \eta^{2} w}{6}(\dot{b} w-b \dot{w})+4 \gamma \eta^{2} w v \\
& +2 \eta^{2} w\left(v^{2}+\frac{1}{3 w^{2}}+\frac{\pi^{2} b^{2}}{12}\right)+\frac{4 g \eta^{4} w}{3}+4 \pi \Gamma \eta^{2} w G,
\end{aligned}
$$

where the coupling function $G=G(\xi, \zeta, w)$, written as function of the auxiliary variables $\xi=2 p / w$ and $\zeta=$ 

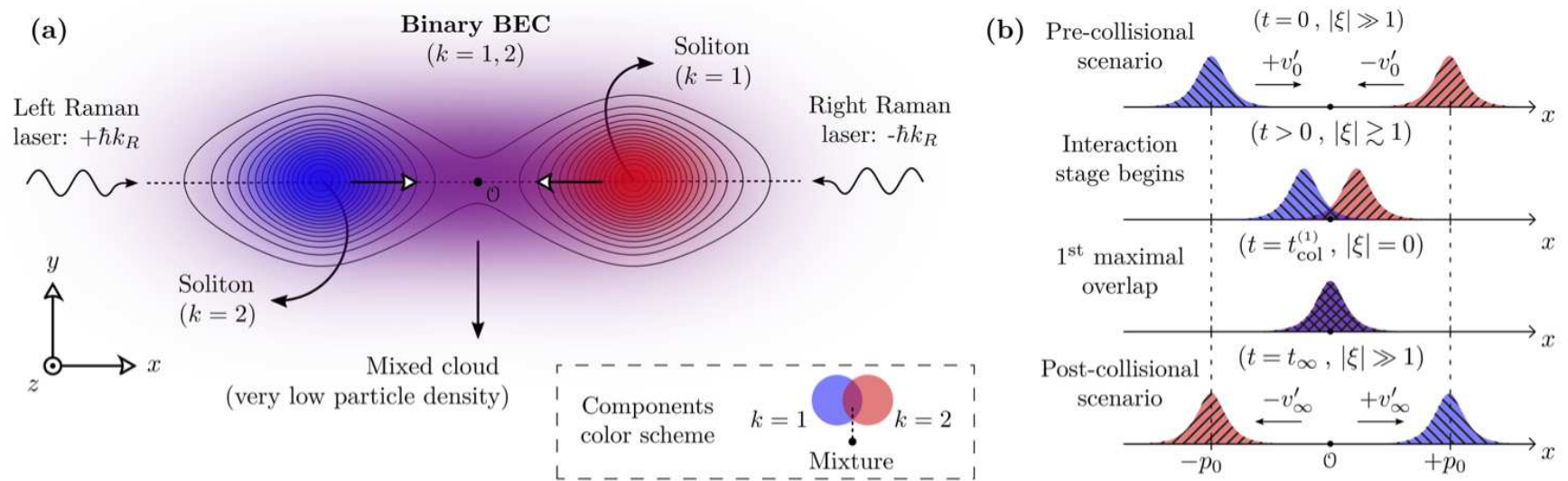

FIG. 1. (Color online) Pictorial representation of the pre-collisional scenario of two symmetric solitons in a SO and Rabi-coupled $\mathrm{BEC}$. In (a) and in the top frame of (b), the pre-collisional scenario consists of both solitons (initially with peak position at $x= \pm p_{0}$ ) moving toward the origin $(\mathcal{O})$ with propagation velocity ${\overrightarrow{v_{0}}}^{\prime}=(-1)^{k}\left(v_{0}+\gamma\right) \hat{x}$ (for $k=1,2$ and $v_{0}^{\prime}=v_{0}+\gamma>0$ ), for the $k$ soliton component, that is induced by the initial phase velocity and by the Raman laser field pumped in the $(-1)^{k} \hat{x}$ direction. The remaining three frames in (b) illustrate the evolution of the initial configuration, i.e., by showing the beginning of the interaction stage, which is followed by the first collision process with maximum overlap at $t=t_{\mathrm{col}}^{(1)}$. The last frame depicts the post-collisional scenario, with the scattered solitons moving away from each other with propagation velocity $v_{\infty}^{\prime}$ and eventually reaching their initial separation at $t=t_{\infty}$.

$2 v+\xi b$ plus the parameter $w$, is given by

$$
G(\xi, \zeta, w)=\frac{\sin (\zeta p)}{\sinh (\xi) \sinh (\pi \zeta w / 2)}
$$

Since the resulting Lagrangian depends upon the global phase only through the term $\left(4 \eta^{2} w\right) \dot{\sigma}$, the EulerLagrange equation for $\sigma$ provides the norm conservation in the reduced ODE model, i.e.,

$$
K=4 \eta^{2} w,
$$

which simply states that $\int_{-\infty}^{\infty} d x\left(\left|A_{+}\right|^{2}+\left|A_{-}\right|^{2}\right)=K$, allowing one to acquire $\eta(t)$ directly from $w(t)$. Also, the other Euler-Lagrange equations arising from the Lagrangian (4) yield a system of four coupled ODEs, the so-called reduced model, written as

$$
\begin{gathered}
\dot{v}=\pi \Gamma \frac{\partial G}{\partial p}, \\
\dot{w}=b+\frac{12 \Gamma}{\pi} \frac{\partial G}{\partial b} \\
\dot{p}=-\left(v^{\prime}+\pi \Gamma \frac{\partial G}{\partial v}\right), \\
\dot{b}=\frac{3}{\pi^{2}}\left(\frac{4}{3 w^{3}}+\frac{g K}{3 w^{2}}-4 \pi \Gamma \frac{\partial G}{\partial w}\right),
\end{gathered}
$$

with $v^{\prime}=v+\gamma$. These equations govern the evolution of the four independent variational parameters that characterize the system of symmetric solitons possessing the fixed functional form given by the ansatz (2).

The set of parameters $\mathcal{C}(t)=\{p(t), v(t), w(t), b(t)\}$ expresses the configuration of the system at an instant of time $t>0$, which evolves from an initial configuration $\mathcal{C}_{0}=\left\{p_{0}, v_{0}, w_{0}, b_{0}\right\}$ (here we use the notation: $\left.q(0)=q_{0}\right)$. To properly investigate the scattering of symmetric solitons in this variational model, one needs to build a set of $\mathcal{C}_{0}$ that corresponds to a desired precollisional scenario. In Fig. 1, two illustrative representations of such pre-collisional scenario are shown. In this case, we have $|\xi| \gg 1$, which means that the separation of the solitons (given by $2|p|$ ) is much greater than their width, providing a negligible tail overlap at the origin of the coordinate system, such that the system can be represented by two noninteracting symmetric solitons. This correspondence is no longer valid when the interaction stage begins, i.e., at the "moment" in which the decreasing separation is $|\xi| \gtrsim 1$, and the increasing overlap of the solitons' tails eventually becomes large enough so that the effects of the Rabi interaction becomes substantial.

We will see (next section) that the interacting solitons can collide once or several times. In the latter case, they can form a bound-state that endures until the last collision. Each collision is a process that mostly affect the dynamics of the solitons during the time near the instant of maximal overlap (as depicted in Fig. 1(b) for the first collision), which is denoted by $t=t_{\text {col }}^{(i)}$ for the $j$-th collision (hence, $p\left(t_{\mathrm{col}}^{(j)}\right)=0$ ), with $j=1, \ldots, n_{\mathrm{col}}$ and $n_{\mathrm{col}}$ being the total number of collisions during the boundstate.

These collision processes can induce width oscilla- 
tions in the solitary waves. It is a dynamical property that manifests when a part of the solitons' kinetic energy is contained within a wave profile vibration. Such property plays a very important role in the bound-state dynamics and can prevail after the unbinding. So, one can expect that the post-collisional scenario is characterized by scattered solitons moving away from each other and endowed with width oscillations (this scenario is illustrated in Fig. 1(b) for a transmission case). As their separation gradually increases, the inequality $|\xi| \gg 1$ eventually holds, allowing the noninteracting solitons correspondence to be applied again.

In this work we focus on the scattering of solitary waves manifesting in the form of fundamental soliton solutions during the pre-collisional scenario, this means that the solitons' shape remains practically the same until the interaction stage (no width change: $\dot{w}=0$ ). Width oscillations during the post-collisional scenario are expected and analytically tractable due to the simplifications allowed by the $|\xi| \gg 1$ regime in the reduced model equations (Eqs. (7a)-(7d)). Hence, the width dynamics in this regime is studied in the next section, which also introduces some important concepts and definitions regarding the total energy of the system, which are essential in the discussions concerning the main issue of this article.

\section{INITIAL CONDITIONS AND WIDTH OSCILLATIONS}

In order to build the general form of a set of parameters $\mathcal{C}_{0}$ for pre-collisional scenarios, some basic insight about the solitons' dynamics in the reduced model is required, and hence the Eqs. (7a)-(7d) need to be analyzed. Firstly, note that in all four equations there is a term directly proportional to $\Gamma(\partial G / \partial q)$ (with $q=v, w, p$ or $b)$, which couples the variational parameters with each other. When the solitons are far from each other (as in pre- or post-collisional scenarios), i.e., for $|\xi| \gg 1$, these coupling terms become negligible since the denominator of $G$ increases very fast for large $|\xi|$ due to a dominating term $\propto \exp [-|(1+\pi w b / 2) \xi|]$, allowing one to assume that $\partial_{q} G \approx 0$ and $G \approx 0$. In this regime, the reduced model describes noninteracting solitons (with null acceleration $\dot{v}=0$ in (7a)) moving toward (outward) the origin when $\dot{p}<0(\dot{p}>0)$, with constant absolute velocity $\left|v^{\prime}\right|$ as stated by Eq. (7c). Also, this equation shows that $v$ can be identified as the propagation velocity (given by $\dot{p}$ ) only in the absence of the SO coupling $(\gamma=0)$. Note that the above approximations fails when the solitons get closer to each other, such that the term $\pi \Gamma \partial_{v} G$ becomes relevant. In fact, the role of the variational parameter $v$ consists in emulating the effect of the phase velocity that, together with the group velocity $\gamma$ induced by the SO coupling, promote the collisional scenario of solitons moving initially with propagation velocity $v_{0}^{\prime}=v_{0}+\gamma>0$ (as previously pointed out in Fig. 1).

Eqs. (7b) and (7d) govern the dynamics of the shape parameters $(w, b)$. In the regime $|\xi| \gg 1$, the parameter $b$ dictates the variations in the width, since $b=\dot{w}$, where the conditions for a fixed profile can be derived by simultaneously imposing $b=0$ and $\dot{b}=0$. The solutions are $w_{f}=4 /(|g| K)$ and $b_{f}=0$, with $f$ standing for fundamental (without oscillation). Then, to get a pre-collisional configuration consisting of fundamental solitons, one can simply use a set of initial parameters in the form $\mathcal{C}_{0}^{f}=\left\{p_{0}, v_{0}, w_{f}, b_{f}\right\}$ such that $v_{0}>-\gamma$ and $\left|\xi_{0}\right| \gg 1$. Next, by considering slightly different shape parameters, an analytical study of the width behavior can be directly performed by means of the dynamic equations. To this end, the width parameter must be rewritten as $w(t)=[1+W(t)] w_{f}$, with the new parameter $W(t) \ll 1$ being the relative deviation from $w_{f}$. The latter assumption allows one to expand the Eq. (7d) in Taylor series $\left((1+W)^{-n}=1-n W+\mathcal{O}\left(W^{2}\right)\right.$ for $n>0)$, in view to find the following equations:

$$
\left\{\begin{array}{l}
w_{f} \dot{W}-b=0 \\
W+\dot{b} / \mathcal{B}=0
\end{array} \quad, \quad \mathcal{B}=\frac{4}{\pi^{2} w_{f}^{3}}\right.
$$

neglecting terms of order $\mathcal{O}\left(W^{2}\right)$. The equations ((8)) can be cast in a decoupled form $\ddot{q}+\left(\mathcal{B} / w_{f}\right) q=0$ (with $q=W$ or $b$ ), which reveals that both $w$ and $b$ undergo harmonic oscillations with angular frequency $\omega_{w}^{(\mathrm{LO})}=\sqrt{\mathcal{B} / w_{f}}=g^{2} K^{2} /(8 \pi)$ (LO stands for low amplitude oscillations). Additionally, Eqs. (8) show that these parameters oscillate out of phase by $\pi / 2$ radians with oscillation amplitudes $\hat{W}$ and $\hat{b}$ related through the ratio $\hat{b} / \hat{W}=\omega_{w}^{(\mathrm{LO})}$, hence the condition $\hat{W} \ll 1$ implies in $\hat{b} \ll 1$.

In the interaction stage, the coupling terms containing $\partial_{q} G$ influence the system's dynamics in a nontrivial way that cannot be analytically tractable. Since the shape parameters are altered during the collision processes, width oscillations are expected to occur, but the behavior is far from being quasi-harmonic because the inequality $|\xi| \gg 1$ does not hold and $\hat{W}$ is not small. The latter condition also applies to the post-collisional scenarios, i.e., the scattered solitons can be provided with highly nonharmonic width oscillations. To investigate this case, one can explore the fact that total energy of the system is a conserved quantity, given by the Hamiltonian

$$
H(p, v, w, b)=H_{\mathrm{TM}}+H_{\mathrm{VM}}+\pi \Gamma\left(G-G_{0}\right),
$$


where

$$
\begin{aligned}
& H_{\mathrm{TM}}(p, v)=\frac{1}{2}(v+\gamma)^{2}+\pi \Gamma G_{0}, \\
& H_{\mathrm{VM}}(w, b)=\frac{K g}{12 w}+\frac{1}{6 w^{2}}+\frac{\pi^{2}}{24} b^{2}, \\
& G_{0}=\left.G\right|_{(w, b)=\left(w_{f}, 0\right)}=\frac{\sin (2 p v)}{\sinh \left(2 p / w_{f}\right) \sinh \left(\pi v w_{f}\right)} .
\end{aligned}
$$

The first and the second terms in the Hamiltonian correspond to the energy within the solitons' translational mode (TM) and vibrational mode (VM), respectively, and the third is an energy term due to the interaction of these modes [72]. The idea of casting the Hamiltonian as shown in (9) is to highlight the energy contributions arising from each type of motion of the solitons in the reduced model.

The Hamiltonian (9) in its entire form will be used in the next section. For a while, the focus is on the general behavior of width oscillations emerging in postcollisional scenarios. In this sense, terms originating from the function $G$ are negligible, allowing one to identify the solitons' TM energy by their kinetic energy, i.e., $H_{\mathrm{TM}}\left(v^{\prime}\right)=\left(v^{\prime}\right)^{2} / 2$. By considering the configurations at $t=0$, given by $\mathcal{C}_{0}^{f}$, one obtains the Hamiltonian

$$
H_{0}\left(v_{0}\right)=H_{\mathrm{TM}}^{(0)}+H_{\mathrm{VM}}^{(0)},
$$

where the first term, $H_{\mathrm{TM}}^{(0)}=H_{\mathrm{TM}}\left(v_{0}^{\prime}\right)$, is the TM initial energy, and the last, $H_{\mathrm{VM}}^{(0)}=H_{\mathrm{VM}}\left(w_{0}, b_{0}\right)=-g^{2} K^{2} / 96$, is the self-energy of the fundamental solitons. After an "infinitely" long time interval $(t \rightarrow \infty)$, the Hamiltonian of the scattered solitons can be written as

$$
H^{(\infty)}=H_{\mathrm{TM}}^{(\infty)}+H_{\mathrm{vM}}^{(\infty)},
$$

where $H_{\mathrm{TM}}^{(\infty)}=H_{\mathrm{TM}}\left(v_{\infty}^{\prime}\right)$, with $v_{\infty}=v(t \rightarrow \infty)$, and

$$
H_{\mathrm{VM}}^{(\infty)}=\left.\left(\frac{K g}{12 w}+\frac{1}{6 w^{2}}+\frac{\pi^{2} b^{2}}{24}\right)\right|_{t \rightarrow \infty} .
$$

Here, $v_{\infty}$ is the final (constant) value of the phase velocity, and $H_{\mathrm{TM}(\mathrm{VM})}^{(\infty)}$ is the TM (VM) final energy. We stress that the parameter $v$ approaches $v_{\infty}$ asymptotically during the post-collisional scenario, but in a practical sense, one can set $t_{\infty}$ as the instant in which the initial separation is reattained $\left(p\left(t_{\infty}\right)=p_{0}\right)$, where $t \rightarrow \infty$ in (11) was replaced by $t=t_{\infty}$ (as shown in the last frame in Fig. $1(\mathrm{~b}))$.

The energy conservation implies that $\Delta H=H^{(\infty)}-$ $H^{(0)}=0$. By using this result combined with the equations $b=w$ and $w=(1+W) w_{f}$, one can obtain the following equation for the parameter $W(t)\left(t>t_{\infty}\right)$ in terms of the initial and final propagation velocities

$$
(\pi \dot{W})^{2}+\left(\frac{g^{4} K^{4}}{64}\right) \frac{W^{2}}{(1+W)^{2}}=-\left(\frac{3 g^{2} K^{2}}{2}\right) \Delta H_{\mathrm{TM}},
$$

where $\Delta H_{\mathrm{TM}(\mathrm{VM})}=H_{\mathrm{TM}(\mathrm{VM})}^{(\infty)}-H_{\mathrm{TM}(\mathrm{VM})}^{(0)}$ is the TM (VM) energy variation, obeying the relation $\Delta H_{\mathrm{TM}}=-\Delta H_{\mathrm{VM}}$. Based on the positiveness of all terms in the left-hand side of the Eq. (12), the energy variation of the modes are such that $\Delta H_{\mathrm{TM}} \leq 0$ and $\Delta H_{\mathrm{VM}} \geq 0$, which implies $\left|v_{\infty}^{\prime}\right| \leq v_{0}^{\prime}$ (recall that $v_{0}^{\prime}>0$ ) with the equalities holding when the scattered solitons have no vibrational profile $(W=\dot{W}=0)$. Except for this latter trivial case, $W$ has two critical values (denoted by $W_{c}^{ \pm}$) that are obtained from Eq. (12) subjected to the condition $\dot{W}=0$. These critical values are found to be

$$
W_{c}^{ \pm}= \pm \frac{\sqrt{6 \Delta H_{\mathrm{VM}}}}{|g| K / 4 \mp \sqrt{6 \Delta H_{\mathrm{VM}}}},
$$

with $W_{c}^{+} \geq 0$ being the positive critical value and $W_{c}^{-} \leq 0$ the negative one. In view to solve the first order differential equation for $W$, one gets

$$
d t=\frac{8 \pi}{g K} \frac{(1+W) d W}{\sqrt{96 \Delta H_{\mathrm{VM}}(1+W)^{2}-g^{2} K^{2} W^{2}}} .
$$

Indeed, it appears to be a hard task to solve Eq. (14) for $W(t)$. However, the behavior of the width parameter is periodic. So, one can write $t\left(W_{c}^{+}\right)-t\left(W_{c}^{-}\right)$equal to half of the width oscillation period $\left(T_{w} / 2\right)$. Hence, by using the relation $\omega_{w}=2 \pi / T_{w}$, the angular frequency of width oscillations is found to be

$$
\omega_{w}=\omega_{w}^{(\mathrm{LO})}\left[1-\frac{\Delta H_{\mathrm{VM}}}{\left|H_{\mathrm{VM}}^{(0)}\right|}\right]^{3 / 2}, \quad \Delta H_{\mathrm{VM}} \leq\left|H_{\mathrm{vM}}^{(0)}\right| .
$$

The Eqs. (13) and (15) characterize the width oscillations in the post-collisional scenario in terms of the initial and final propagation velocities, $v_{0}^{\prime}$ and $v_{\infty}^{\prime}$, which provide the energy increase in the VM $\left(\Delta H_{\mathrm{vM}}=\left[\left(v_{0}^{\prime}\right)^{2}-\left(v_{\infty}^{\prime}\right)^{2}\right] / 2\right)$. Since $\left|v_{\infty}^{\prime}\right| \leq v_{0}^{\prime}$, the scattering can be of three types, namely, elastic (case $\left|v_{\infty}^{\prime}\right|=v_{0}^{\prime}$ ), inelastic (case $\left|v_{\infty}^{\prime}\right|<v_{0}^{\prime}$ ), and completely inelastic (case $\left|v_{\infty}^{\prime}\right|=0$ ). An elastic scattering occurs when the TM energy is completely recovered after the interaction stage, resulting in scattered solitons with fixed shape $\left(\Delta H_{\mathrm{VM}}=0\right.$ and $\left.W_{c}^{ \pm}=0\right)$, otherwise the amount of energy not recovered remains stored in the VM (inelastic scattering), and the scattered solitons will vibrate $\left(\Delta H_{\mathrm{VM}}>0\right.$ and $\left.W_{c}^{ \pm} \neq 0\right)$. If this amount of energy is very small such that $\left|v_{\infty}^{\prime}\right| \lesssim v_{0}^{\prime}$ (quasi-elastic scattering), the vibration can be considered to be quasi-harmonic because $\Delta H_{\mathrm{VM}} \ll\left|H_{\mathrm{VM}}^{(0)}\right|$ implies that $W_{c}^{+} \approx\left|W_{c}^{-}\right| \ll 1$ and $\omega_{w} \approx \omega_{w}^{(\mathrm{LO})}$, which validate the results of the previous approach for low amplitude of width oscillations. If the TM final energy is zero, the total energy of the system is entirely contained in the VM (completely inelastic scattering, $\Delta H_{\mathrm{VM}}=\left(v_{0}^{\prime}\right)^{2} / 2$ or $\left.H^{(\infty)}=H_{\mathrm{VM}}^{(\infty)}\right)$, resulting in scattered solitons with fixed separation vibrating with the largest (lowest) possible amplitude (frequency). In 
terms of width oscillations, this means that for a specific value of $v_{0}^{\prime}$, the critical values of $|W|$ are maximum and $\omega_{w}$ is minimum.

Since the knowledge about $v_{\infty}^{\prime}$ it is enough for us to characterize both TM and VM dynamics of the scattered solitons, the investigation of solitons' scattering starts from the choice of initial value of $v_{0}^{\prime}$ and its influence over the interaction stage.

\section{NUMERICAL RESULTS AND DISCUSSION}

We set the value of nonlinearity strength $g$ such that the width of the fundamental soliton solution is $w_{f}=1$ and the solitons' total norm $K=1$. These constraints are attained for $g=-4$. Also, we set the Rabi coupling as $\Gamma=-0.04$, which allow us to get interesting dynamical effects. The interaction between the solitons is sufficiently small for a 20 units wide separation, which justify our choice of $p_{0}=10$. The program developed for the simulations uses double precision for both real and complex numbers, it is written in the Fortran 95 language and employs a $4^{\text {th }}$-order Runge-Kutta method to numerically solve the coupled ODEs (7a)-(7d) with initial conditions given by $\mathcal{C}_{0}^{f}\left(v_{0}^{\prime}\right)$ and $v_{0}^{\prime}=v_{0}+\gamma>0$ being a variable initial parameter defining the pre-collisional configuration. The time-step is set to $10^{-4}$, this value is small enough to provide a very good approximation for the evolution of the variational parameters in the conditions of our interest. Also, in order to check the accuracy of the results obtained, we performed some tests by considering lower values of discretization, for which we obtained similar results.

To explore the influence of $v_{0}^{\prime}$ over the solitons' dynamics, an iterative routine is implemented to perform a set of consecutive scattering simulations, each one using a different initial propagation velocity, $v_{0}^{\prime}(j)$ (with $j \in \mathbb{N}$ being the iteration number), which can only assume values within a predefined $v_{0}^{\prime}$-range $\left[v_{0}^{\prime}(1), v_{0}^{\prime}\left(n_{I}\right)\right]$ (with $n_{I}$ being the total number of iterations). In this routine, the value of the SO coupling constant $\gamma$ is kept fixed while $v_{0}$ is increased by a fixed amount $\delta v_{0}>0$ in the end of each iteration, i.e., $v_{0}^{\prime}(j+1)=v_{0}^{\prime}(j)+\delta v_{0}$. The length of the continuous interval defined by the $v_{0}^{\prime}$-range is simply given by the difference between the $v_{0}^{\prime}$ values used in the first and in the last scattering simulation, $L=v_{0}^{\prime}\left(n_{I}\right)-v_{0}^{\prime}(1)$, and consequently $\delta v_{0}=L /\left(n_{I}-1\right)$. Moreover, for each scattering simulation the output data is obtained when the numerical evolution stops after the program detects that the initial separation was reached in the post-collisional scenario. In this sense, the quantities analyzed are the number of collisions before unbinding $n_{\mathrm{col}}$, the exit velocity $v_{\infty}, W_{c}^{+}$, and $T_{w}$.

We stress that we choose a convenient integer $n_{\text {ref }}$ as reference and only the points with $n_{\text {ref }}-4 \leq n_{\text {col }} \leq$ $n_{\text {ref }}-1$ are considered in our graphical analyzes. So, the remaining points, with $n_{\mathrm{col}}<n_{\mathrm{ref}}-4$ or $n_{\mathrm{col}}>n_{\text {ref, }}$ are not plotted.

\section{A. Scattering process without SO coupling $(\gamma=0)$}

In this subsection we will consider the system in absence of SO coupling $(\gamma=0)$. This first step will provide us a reference for the dynamical properties, which will be analyzed in details in order to verify, in the next subsection, the influence of the SO coupling parameter $\gamma$ over them.

The results of the iterative simulations show that in the high-energy collision regime $\left(v_{0} \gg 1\right)$ the solitons collide one time $\left(n_{\mathrm{col}}=1\right)$ and their phase velocity almost does not diminishes $\left(v_{\infty} \lesssim v_{0}\right)$, indicating that the scattering is quasi-elastic and that the single collision process promotes just a direct transmission (the solitons simply pass through each other). In this regime, as $v_{0}$ increases the quantities $v_{\infty}, W_{c}^{+}$, and $\omega_{w}$ asymptotically approach the lines $v_{\infty}=v_{0}, W_{c}^{+}=0$, and $\omega_{w}=\omega_{w}^{(\mathrm{LO})}=2 / \pi$, respectively, which are associated with the "scattering" of two noninteracting symmetric solitons. As $v_{0}$ is reduced, the scattering gradually becomes more inelastic, that is, $\Delta H_{\mathrm{VM}}$ increases causing $W_{c}^{+}$to increase too and $\omega_{w}$ to decrease. When $v_{0}$ is close to the value $v_{(\mathrm{VM})}=0.374$, the excitation of the vibrational mode is maximum although the variation in the translational mode energy is still relatively small (since $v_{\infty} \approx 0.898 v_{0}$ ), this means that $W_{c}^{+}$is maximum too and $\omega_{w}$ is minimum, with $\max _{G}\left(W_{c}^{+}\right) \approx 0.397$ and $\min _{G}\left(\omega_{w}\right) \approx 0.881 \omega_{w}^{(\mathrm{L})}$ (the estimates were obtained from graphical analyses, and $G$ stands for global, i.e., for any $v_{0}>0$ ). Accordingly, as $v_{0}$ gets even smaller (low-energy collision regime $v_{0}<v_{(\mathrm{VM})}$ ), $\Delta H_{\mathrm{VM}}$ and, consequently, $W_{c}^{+}$decreases too (the opposite stands for $\left.\omega_{w w}\right)$. The origin of this inversion in the behavior of these quantities can be understood by analyzing the equation $\Delta H_{\mathrm{VM}}=\left(v_{0}-v_{\infty}\right)\left(v_{0}+v_{\infty}\right) / 2$ for decreasing $v_{0}$. The first factor always grows because the scattering becomes more inelastic, and it dominates during the highenergy collision regime. On the contrary, the second factor always declines due the reducing amount of energy involved in the first collision, it exactly balances the growth promoted by the first one when $v_{0}=v_{(\mathrm{VM})}$, and dominates during the low-energy collision regime causing $\Delta H_{\mathrm{VM}}$ to decrease. This behavior persists until $v_{0}$ reaches a critical value $v_{c} \approx 0.11755$, that corresponds to a completely inelastic scattering $\left(v_{\infty}=0\right)$. If $v_{0}<v_{c}$, the solitons form a bound-state after the first collision process and $n_{\text {col }} \geq 2$. The scattering simulations in this range reveal that the dynamics of this bound-state is very complex and rich in details, requiring a quite extensive investigation in order to understand the underlying mechanism produced by the attractive Rabi interaction. From hereafter, the focus is on the correlations between the output quantities and the control (input) parameter $v_{0}^{\prime} \in\left(0, v_{c}^{\prime}\right)$, and how these arise from the reduced model description of the solitons' bound-state. 

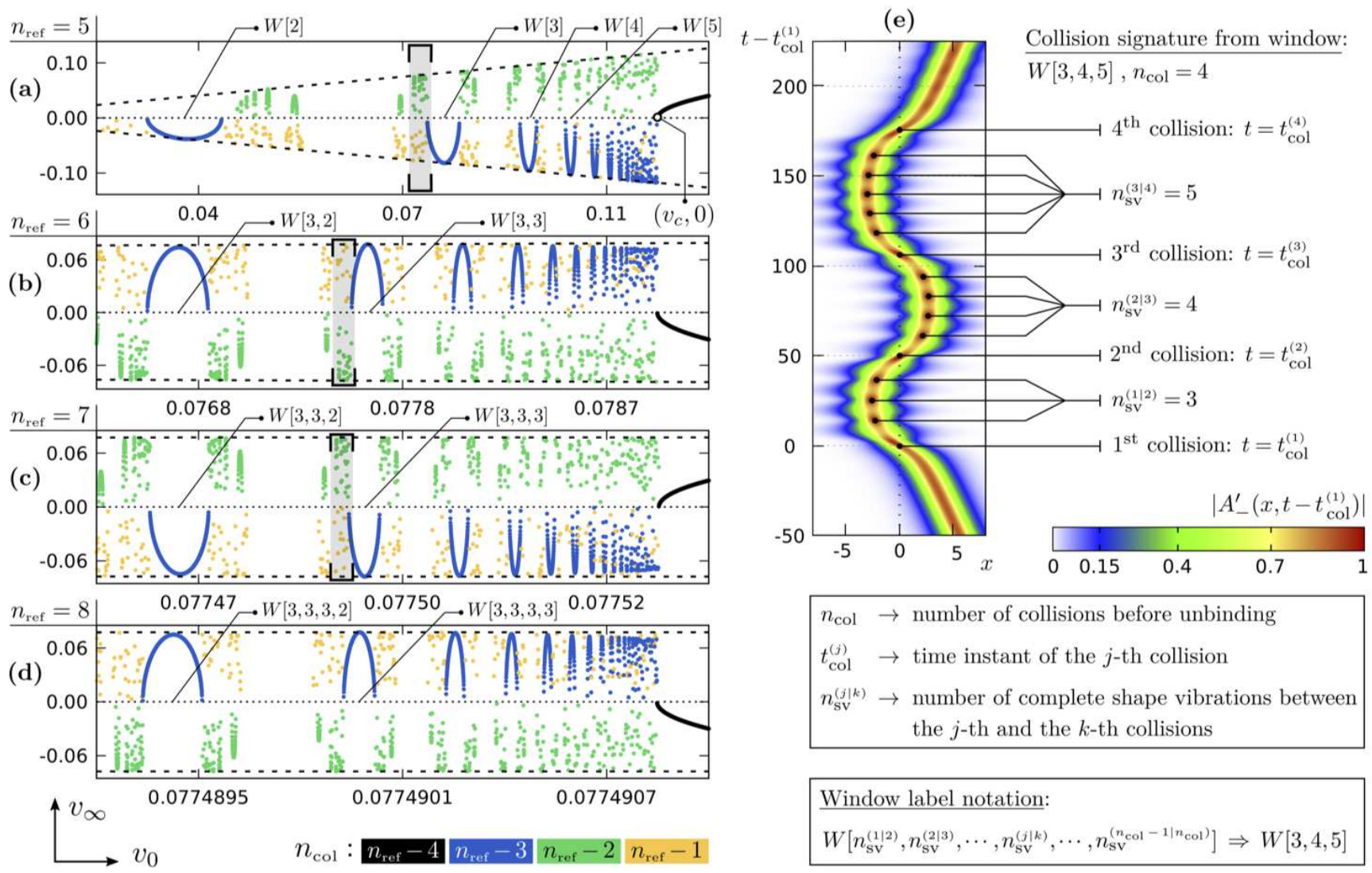

\begin{tabular}{|l|}
\hline Window label notation: \\
$W\left[n_{\mathrm{sv}}^{(1 \mid 2)}, n_{\mathrm{sv}}^{(2 \mid 3)}, \cdots, n_{\mathrm{sv}}^{(j \mid k)}, \cdots, n_{\mathrm{sv}}^{\left(n_{\mathrm{col}}-1 \mid n_{\mathrm{col}}\right)}\right] \Rightarrow W[3,4,5]$
\end{tabular}

FIG. 2. (Color online) Left panel: Scattering results for $v_{\infty}$ versus $v_{0}$ obtained via iterative simulations of the reduced the ODE model (Eqs. (7a)-(7d)) in four $v_{0}$-ranges (a)-(d), within the interval $\left[0, v_{c}\right]$ and with $\gamma=0$, i.e., without the SO coupling effect. The highlighted rectangular regions (gray) indicate the $v_{0}$-range of the plot immediately below, i.e., the panels in (b), (c) and (d) are successive "zooms" of the highlighted regions. The color scheme at the bottom of this figure uses the integer $n_{\text {ref }}$, called reference number (its value is specified in top right corner of each plot), to provide an adaptive rule for coloring the points $\left(v_{0}, v_{\infty}\right)$ accordingly to the integer $n_{\text {col }}$ associated with the corresponding regular process (irregular ones are not plotted since $\left.n_{\text {col }} \gg 1\right)$. Also, some windows are labeled in each plot, where the used notations are explained in the right panel. Right panel: (e) heatmap of the normalized function $\left|A_{-}^{\prime}(x, t)\right|$. The $v_{0}$ value used in this simulation belongs to the interval of regularity of a 4-pass collisional scattering window. The notation used in the description of this heatmap is explained in the bottom boxes of this panel.

In Fig. 2, the left panel shows four plots of $v_{\infty} \times v_{0}$, which were generated from the data provided by the iterative simulations. Specifically, the panel (a) (with $n_{\text {ref }}=5$ ) covers a $v_{0}$-range in the low-energy collision regime, where $\left(v_{c}, 0\right)$ can be seen as a critical-point that separates the region of direct transmission, or 1-pass collisional scattering (points with $n_{\mathrm{col}}=n_{\text {ref }}-4=1$, see the color scheme at the bottom of the figure), from the region of multi-pass collisional scattering, where the postcollisional scenario is always preceded by the formation of a bound-state (points with $n_{\text {col }}=2,3$ and 4 ). The distribution of points in this plot reveals that $v_{\infty}$ and $n_{\text {col }}$ obey the equation $\operatorname{sign}\left(v_{\infty}\right)=(-1)^{n_{\mathrm{col}}-1}$, which states that a transmission-like scattering $\left(v_{\infty}>0\right)$ always occurs when $n_{\text {col }}$ is odd, and a reflection-like scattering $\left(v_{\infty}<0\right)$ always occurs when $n_{\text {col }}$ is even.

Regarding the region of 1-pass collisional scattering (Fig. 2(a)), one can verify that the points $\left(v_{0}, v_{\infty}\right)$ closely trace the upper segment of a hyperbola with functional form $x^{m}-y^{m}=v_{c}^{m}$ (with $v_{0}$ and $v_{\infty}$ taking the roles of $x$ and $y$, respectively), which has its vertex in the critical point and its asymptotes (the lines $y= \pm x$ ) represented by the dashed lines in Fig. 2. Next, by a fitting procedure we get $m=1.814 \pm 0.007$, showing that the collision outcome can be predicted very accurately when $v_{0} \geq v_{\mathcal{c}}$. This control is possible because a small variation in the initial velocity $v_{0} \rightarrow v_{0}+\delta$, causes a small variation in its final velocity $v_{\infty} \rightarrow v_{\infty}+\Delta$, with $\delta$ and $\Delta$ having the same order of magnitude, and the scattering is said to be regular in this sense. On the other hand, the same does not hold when $v_{0}<v_{c}$, since $v_{\infty}$ is found to be very sensitive to small changes in the values $v_{0}$ for some regions. Indeed, there are some regions with regularity for $v_{0}<v_{c}$, in which we can obtain predictable results. The most evident intervals of regularity are those where only 2-pass collision scattering $\left(n_{\text {col }}=2\right)$ happens, called reflection windows, which are seen as valley-like shapes in Fig. 2(a). The asymptote $y=-x$ is tangent to the 
curve defined by all these shapes, which means that an elastic 2-pass collision scattering is possible for a specific $v_{0}$ value within each reflection window. Interestingly, these windows appear to form a structure that presents self-similarity at any scale (a fractal-like scattering), i.e., any amplification of a smaller $v_{0}$-range containing the critical point reveals (given an enough point density) the same pattern of infinitely many reflection windows intertwined by regions in which $n_{\text {col }}>2$. This happens because both the length of a window and its separation distance to the nearest window can become arbitrarily small as close as it gets to the critical point.

Regarding 3-pass collisional scattering $\left(n_{\mathrm{col}}=3\right)$, the Fig. 2(a) shows that it can happen if the $v_{0}$ value is sufficiently close to one of the edges of any reflection window, where some of the associated points are found to be within very small intervals of regularity, which technically requires a much higher local point density to be reasonably visualized. Therefore, in order to verify how these points are really distributed, iterative simulations were performed in $v_{0}$-ranges near the left and right sides of certain reflection windows. The complementary data acquired unfolds some substructures of transmission windows that were previously hard to detect, and strongly indicate that 3-pass collisional scatterings can only occur when $v_{0}$ falls into an interval of regularity corresponding to one of these transmission windows, assuming lump-like shapes in Figs. 2(b). These substructures are endowed with the same self-similarity property previously discussed, but only those emerging at the left side of a reflection window present a pattern that resembles the one shown in the panel (a) (the windows height in right-sided substructures decrease instead of increasing accordingly with the asymptote $y=x$ ). Indeed, the range that encompasses the larger left-sided substructure, highlighted by a rectangular (gray) region in Fig. 2(a), was simulated again with more points and displayed in Fig. 2(b). This plot provides a wide view of the particular substructure chosen in Fig. 2(a), where one can notice that both the window pattern and the distribution of points near the windows edges are indeed very similar ("mirrored") to that of the first plot.

By investigating the surroundings of the transmission windows through some iterative simulations, other smaller substructures associated with 4-pass collisional scatterings are revealed. These are composed by reflection windows too and present a high degree of similarity with the previous plot, a signature of the fractallike scattering, as one can attest by comparing it with the plot in Fig. 2(c), which considers the left-sided substructure of the second transmission window (the highlighted (grey) region in Fig. 2(b)). Thus, all the plotted points within the intervals intertwining the reflection windows in the panel (a) are part of underlying substructures, which unfold whenever one investigates the distribution of points surrounding any reflection or transmission window.
The whole structure composed by infinitely many reflection and transmission windows displays the main characteristic feature of a fractal, i.e., self-similarity. Here, such fractal-like consists of the main window pattern $\left(n_{\mathrm{col}}=2\right)$ plus the left-sided (right-sided) ones associated with $n_{\text {col }}$-pass collisional scatterings $\left(n_{\text {col }} \geq 3\right)$ that emerge in subregions within $v_{0}<v_{\mathcal{c}} \wedge\left|v_{\infty}\right| \leq v_{0}$ that contain only the left (right) critical (or edge) point of a certain $\left(n_{\text {col }}-1\right)$-pass collisional scattering window, which is a point corresponding to a completely inelastic $\left(n_{\text {col }}-1\right)$-pass collisional scattering. A much higher degree of self-similarity is clearly noticed between the window patterns of the substructures, as one can realize by comparing Fig. 2(b) and (c), which appear to be mirrored images (across the $v_{0}$-axis) from each other. The plot displayed in Fig. 2(d) results from iterative simulations in the range highlighted in Fig. 2(c), it emphasizes the fractal feature described and show that the window pattern replicates more precisely in substructures that have the same type of window. As previously mentioned, another feature regarding the solitons' scattering is its high sensitivity to $v_{0}$ when this initial propagation velocity is not within an interval of regularity, this is a signature of chaos that allows us to infer that the scattering is predominantly chaotic when $v_{0}<v_{c}$, which is intrinsically related to the formation of bound-states generally involving a lot of collisions (i.e., $n_{\text {col }} \gg 1$, excepting the region of very low propagation velocities at the left of the larger reflection window). Hence, the fractal structure must arise from a recurrent internal mechanism that causes the scattering to become regular when specific conditions involving the solitons' translational and vibrational modes are attained. We stress that the fractal scattering of solitons of systems described by (generalized) nonlinear Schrödinger equation were also verified in Refs. [55, 58, 59, 62].

To unravel this internal mechanism, a detailed analysis of the solitons' dynamics during the interaction stage is needed. To this end, we first study the general aspects of the bound-states by examining the evolution of the solitons' profile from the perspective of the heatmaps of $\left|A_{-}^{\prime}(x, t)\right|$. For that, several simulations are performed for different values of $v_{0}$ selected in some intervals of those reflection and transmission windows shown in Fig. 2(a)-(d). By analyzing the bound-state formation for various input velocities within a same interval, one can only differ one scattering from another by comparing the shape vibrations and the exit angle $\left(\tan ^{-1}\left(v_{\infty}\right)\right)$ in the post-collisional scenario, that is, before the final collision the dynamics is visually indistinguishable (this is more prominent when considering smaller windows). This means that each window has its own bound-state signature describing the consistent behavior of the solitons' modes that gives rise to the window itself. Moreover, this signature is unique and can be simply defined in terms of the number of complete shape vibrations (a full width oscillation period) between two consecutive collisions during the bound-state, as indicated in Fig. 
2(e). This full width oscillation period is taken as a time interval centered in an instant $t=t_{\text {peak }}$ of minimum profile width (or maximum profile amplitude). In this way one can count the number of peaks (spots in the heatmap where $\left.\left|A_{-}^{\prime}(x, t)\right| \lesssim 1\right)$ between the $(j-1)$-th and the $j$-th collisions $\left(j=2, \ldots, n_{\text {col }}\right)$ and assign the resulting integer value to $n_{\mathrm{Sv}}^{(j-1 \mid j)}$ (see the notation introduced in Fig. 2). Then, any $n_{\text {col }}$-pass collisional scattering window can be labeled in terms of these $n_{\text {col }}-1$ integers as pointed out by Fig. 2(e), where the heatmap displayed corresponds to the 4-pass collisional scattering window $W[3,4,5]$.

Interestingly, the window signatures also follow a pattern that is naturally connected with the fractal-like structure. It is first seen in the panel (a), where the label of the $j$-th window (always from left to right) is written as $W[j+1]$, i.e., $n_{\mathrm{sv}}^{(1 \mid 2)}=j+1$. Then, based on the consistent window patterns previously discussed, one can infer from the heatmaps analysis that the $k$-th window of the substructure emerging from the left side of $W[j+1]$ can be labeled as $W[j+1, k+1]$ (see Fig. 2(b)), with the changing index $J=k+1$ defined as the main index. The same applies for the $l$-th window of the substructure emerging from the left side of $W[j+1, k+1]$, which has the label $W[j+1, k+1, l+1](J=l+1$ is the main index here), and so on.

The integers $n_{\mathrm{sv}}^{(j-1 \mid j)}\left(j=2, \ldots, n_{\mathrm{col}}\right)$ that define a $n_{\mathrm{col}}$ pass collisional scattering window signature depend on the frequency of the shape vibration $\left(\omega_{\mathrm{SV}}\right)$ and on the time duration of each bounce $\Delta t_{\text {bounce }}^{(j-1 \mid j)}=t_{\mathrm{col}}^{(j)}-t_{\mathrm{col}}^{(j-1)}$. In analyzing the shape parameters evolution, we verified that $\omega_{\mathrm{SV}}$ is approximately constant during the bouncing time intervals between collisions, when the tail overlap is small enough so that the interaction promotes an effective attraction maintaining the solitons' bound-state while exerting a weak influence over the previously induced shape oscillations. Also, we found that the quantity $\Delta t_{\text {bounce }}^{\left(n_{\text {col }}-1 \mid n_{\text {col }}\right)}$ (time duration of the last bounce) strictly increases with $v_{0}$ as it covers the entire interval (from left to right) of a $n_{\mathrm{col}}$-pass collisional scattering window (sub)structure, with the corresponding critical point being a singularity in which $\Delta t_{\text {bounce }}^{\left(n_{\text {col }}-1 \mid n_{\text {col }}\right)} \rightarrow \infty$.

If $v_{0}$ is within the interval of regularity of a window with main index $J$, i.e., $n_{\mathrm{sv}}^{\left(n_{\mathrm{col}}-1 \mid n_{\mathrm{col}}\right)}=J$, one can write $\Delta t_{\text {bounce }}^{\left(n_{\mathrm{col}}-1 \mid n_{\mathrm{col}}\right)}=J T_{\mathrm{sv}}+\delta t_{\mathrm{col}}$, in which $T_{\mathrm{sv}}=2 \pi / \omega_{\mathrm{sv}}$ is the shape vibration period and $\delta t_{\mathrm{col}}$ is a $v_{0}$ dependent term accounting for the time duration associated with the $\left(n_{\mathrm{col}}-1\right)$-th and $n_{\mathrm{col}}$-th collisions when $\omega_{\mathrm{sv}}$ is no longer constant. We found that this linear behavior for $\Delta t_{\text {bounce }}^{\left(n_{\mathrm{col}}-1 \mid n_{\mathrm{col}}\right)}$ as function of $J$ occurs when the left or the right edge points of five consecutive windows $(J=1, \ldots, 5)$ are considered. In this case, $\delta t_{\text {col }}$ tends to assume the same value when $v_{0}$ is about to leave the intervals of regularity. The angular coefficient of the fitting line provides a reasonable estimate of $T_{\mathrm{SV}}$, which was obtained with standard deviation always less than $2 \%$ for two sets of five points of each plot in Fig. 2. Con- cerning the structure in the panel (a), the average value obtained was $\left\langle T_{\mathrm{sv}}\right\rangle=10.8 \pm 0.2(1,8 \%)$, while for the substructures in the panels (b)-(d) the average values of $T_{\mathrm{sv}}$ are the same, given by $\left\langle T_{\mathrm{sv}}\right\rangle=9.98 \pm 0.02(0.21 \%)$. The numerical quantity $2 \pi /\left\langle T_{\mathrm{sv}}\right\rangle \approx 0.63$ is a reasonable estimate for the shape vibration frequency, which indicates that such vibrational motion in regular processes have indeed a characteristic frequency.

Next, we analyze the behavior of $\delta t_{\mathrm{col}}$ in terms of $v_{0}$. We found that this quantity strictly increases with $v_{0}$ such that $1.7 \lesssim \delta t_{\mathrm{col}} / T_{\mathrm{sv}} \lesssim 2.0$, with the left (right) sided extreme value reached when $v_{0}$ assumes the value corresponding to the left (right) edge of a window. So, it follows that the bouncing frequency $\omega_{\text {bounce }}^{\left(n_{\text {col }}-1 \mid n_{\text {col }}\right)}=2 \pi / \Delta t_{\text {bounce }}^{\left(n_{\text {col }}-1 \mid n_{\text {col }}\right)}$ must approximately satisfy the relation

$$
(J+1.85+d) \omega_{\text {bounce }}^{\left(n_{\mathrm{col}}-1 \mid n_{\mathrm{col}}\right)}=\omega_{\mathrm{Sv}} \quad(|d| \lesssim 0.15),
$$

which establishes the condition of motion synchronization involving the solitons' translational and vibrational modes, which give rise to the intervals of regularity. This condition means that the bouncing motion is such that $\omega_{\text {bounce }}^{\left(n_{\text {col }}-1 \mid n_{\text {col }}\right)}$ must approach a state of resonance with the shape vibration, $\omega_{\text {bounce }}^{\left(n_{\mathrm{col}}-1 \mid n_{\mathrm{col}}\right)}=\omega_{\mathrm{sv}} /(J+3)$, from below by a suitable amount provided by Eq. (16). The process is always irregular if $\omega_{\text {bounce }}^{\left(n_{\text {col }}-1 \mid n_{\text {col }}\right)}$ is not close enough to or exceeds a resonance value $\left(\omega_{\mathrm{sv}} / 3, \omega_{\mathrm{sv}} / 4, \omega_{\mathrm{sv}} / 5, \ldots\right)$. The narrowing of the windows of a given structure results from the behavior of $\omega_{\text {bounce }}^{\left(n_{\text {col }}-1 \mid n_{\text {col }}\right)}$ with $v_{0}$, which decreases faster as close as $v_{0}$ is from the corresponding critical value in a such way that the greater the integer $J$ is, smaller is the $v_{0}$-interval in which the condition (16) holds and, consequently, narrower is the window.

From section IV, we bring back the quantities defined in (11) to investigate the scattering mechanism in terms of the energy within the solitons' modes. To this end, we firstly considered 20 distinct scattering processes with $v_{0}$ varying into the interval $\left[v_{c}-\Delta v, v_{c}+\Delta v\right]$ from right to left, with $v_{\mathcal{C}}-\Delta v$ chosen to match the $v_{0}$ value of $W[2]$ 's left edge point. The right half of this interval is in the direct transmission region, i.e., the first 10 processes are regular ones consisting of just one collision. In Fig. 3, the temporal evolution of $H_{\mathrm{TM}}$ is shown for each scattering process, with the index $j \in[1,20]$. We observe that for $j \in[1,10]\left(v_{0}>v_{\mathcal{C}}\right)$, the first collision effectively causes a decrease in the energy of the TM that reaches a stable positive constant value $\left(H_{\mathrm{TM}}^{(\infty)}=\right.$ const. $\left.>0\right)$ as the solitons get far apart from each other. For $j=10$, the associated $v_{0}$ value is very close to $v_{C}$ and $H_{\mathrm{TM}}^{(\infty)} \gtrsim 0$. This is an expected result since the "critical process" $\left(v_{0}=v_{\mathcal{C}}\right)$ must end up with $H_{\mathrm{TM}}^{(\infty)}=0$.

From Fig. 3, we verify that for $j \in[11,20]\left(v_{0}<v_{c}\right)$, $H_{\mathrm{TM}}$ is negative and oscillatory (sometimes reaching the positive range again) until the moment of the last collision $\left(t=t_{\text {col }}^{\left(n_{\text {col }}\right)}\right.$, which is close to the time $t-t_{\text {col }}^{\left(n_{\text {col }}\right)}=40$ 


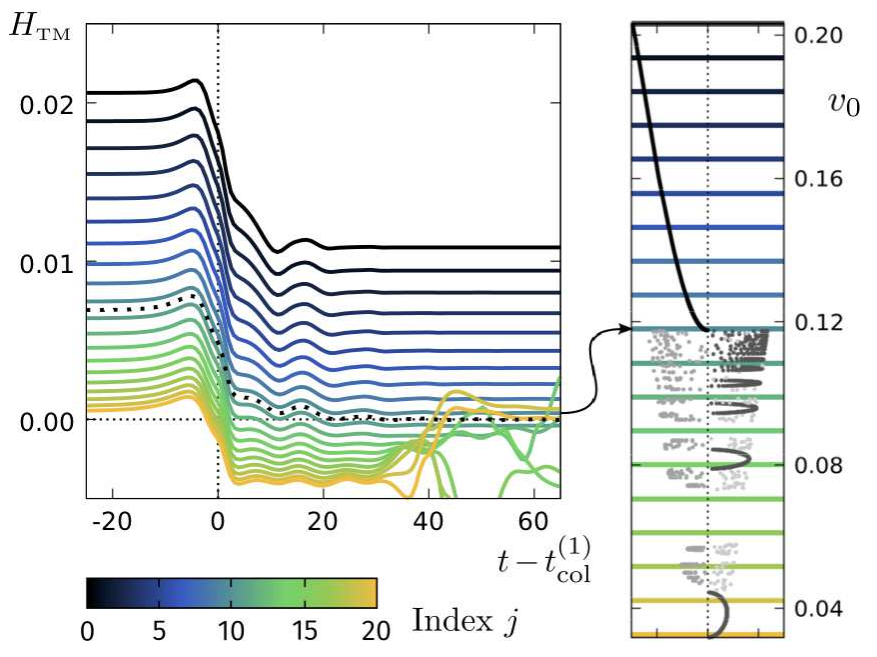

FIG. 3. (Color online) Energy of the solitons' translational mode $\left(H_{\mathrm{TM}}\right)$ as function of the time variable $t-t_{\mathrm{col}}^{(1)}$, by considering 20 scattering processes (indexed with integers $j \in[1,20]$ in the heatmap). The $v_{0}$ values for each one of these processes is highlighted by horizontal lines crossing the rotated version of the plot seen in Fig. 2(a) (right side). The dashed vertical line at the time $t=t_{\mathrm{col}}^{(1)}$ highlights the instant of the first collision, i.e., when the interaction causes an effective decrease in $H_{\mathrm{TM}}$, resulting in part of the initial TM energy converted in VM energy, which in turn promotes shape vibrations. For the 10 last processes $(j=11, \ldots, 20), v_{0}<v_{\mathcal{C}}$ and $H_{\mathrm{TM}}$ becomes negative right after the $t=t_{\mathrm{col}}^{(1)}$.

for the two last processes with $v_{0}$ within $\left.W[2]\right)$. In this case the TM recovers enough energy to remain positive (unbinding) and eventually constant as the separation between the solitons increases. Therefore, these results show that a final negative TM energy value is a signature of the formation of bound-states. Also, during the evolution of this state one can attest that $H_{\mathrm{TM}}$ is indeed a predominantly negative valued function of time, i.e., it can eventually becomes positive valued for a short time without triggering the unbinding and then return to the negative range, but we attested that this can happen only in chaotic processes. Into the regular windows, when $H_{\mathrm{TM}}$ oscillates and reach the positive range, the solitons unbind and scatter away $\left(H_{\mathrm{TM}} \rightarrow H_{\mathrm{TM}}^{(\infty)}>0\right)$.

To clarify the above statement, we proceeded as before by considering 20 distinct scattering processes with $v_{0}$ now covering a full window range. In Fig. 4 , the energy of the solitons' $\mathrm{TM}\left(H_{\mathrm{TM}}\right)$ are shown for $v_{0}$ into the $W[5]$ 's interval of regularity. In 4 (a) we observe that, before the last (second) collision, $H_{\mathrm{TM}}$ is not affected by changes in $v_{0}$. This is because all variational parameter display this same behavior embedded in $H_{\mathrm{TM}(\mathrm{VM})}$, which prevails until the second collision, for which subtle differences accumulated during the bound-state evolution become enough to promote very different interaction outcomes, as one can note in Figs. 4(b) and 4(c). In fact, based on extensive analyses of the simulations data, we were able to infer that this initial dynamics of the modes energy is maintained until the eminence of the last collision for all observed collection of scattering processes within an arbitrary window $W[\ldots, J]$. Also, it extends similarly for any irregular process in the chaotic region nearby, i.e., if the condition of motion synchronization (16) is not met, the solitons do not unbind and any variation in $v_{0}$ causes the upcoming bound-state dynamics to radically diverge, giving rise to the $v_{\infty}$ 's great sensitivity to $v_{0}$.

Following, in Fig. 5 we display the profile $\left|A_{+}^{\prime}\left(x, t-t_{\mathrm{col}}^{(1)}\right)\right|$ and the corresponding $H_{\mathrm{TM}}$ versus $t-t_{\mathrm{col}}^{(1)}$ in order to clarify the basic features regarding both the bound-state and the TM energy dynamics, for each one of the three cases considered in this plot. Note that the heatmap in Fig. 5(a) shows that the collision induce shape vibrations, as also indicated by the corresponding $H_{\mathrm{TM}}$ evolution, where one can see that the TM energy is always positive and $H_{\mathrm{TM}}^{(\infty)}<H_{\mathrm{TM}}^{(0)}$, as expected since part of the initial energy is transferred to the VM. Also, the heatmap shown in Fig. 5(b) is an example of a regular scattering, as previously displayed in Fig. 2(e). This example of regular process is useful for illustrating that the longer the bounce time duration $\left(\Delta t_{\text {bounce }}^{(j-1 \mid j)}\right)$ is, smaller is the absolute value of the TM energy. Indeed, this process occurs because the solitons weakly bind to each other during these well behaved bounces, due to their separation. On the other hand, in irregular processes the bound-state frequently evolves to situations in which the solitons strongly bind to each other, which are characterized by very high bouncing frequencies $\omega_{\text {bounce }}^{(j-1 \mid j)}$ (or collision rates) that maintain the average separation very small. The heatmap from the example in Fig. 5(c) illustrates such behavior. It takes place just after the second collision and is accompanied by a large effective decrease in $H_{\mathrm{TM}}$, which reaches a range of negative values that are greater than $H_{\mathrm{TM}}^{(0)}$ by more than an order of magnitude (in modulus). In fact, one can infer about the binding strength by testing the inequality $\left|H_{\mathrm{TM}}\right| \gg\left|H_{\mathrm{TM}}^{(0)}\right|$, and then infer about the type of scattering process.

\section{B. Effects of SO coupling in the scattering process $(\gamma \neq 0)$}

In the previous subsection, we considered the reduced ODE model in the absence of SO-coupling $(\gamma=0)$, where the results of several scattering simulations revealed the existence of a very rich and complex dynamics that emerges when the initial velocity is smaller than a certain threshold value (i.e., $v_{0}<v_{c}$ ). Our extensive analysis of the data allowed us to better understand the underlying mechanism that gives rise to the many interesting features of the solitons in the variational description. Now we explore what happens 

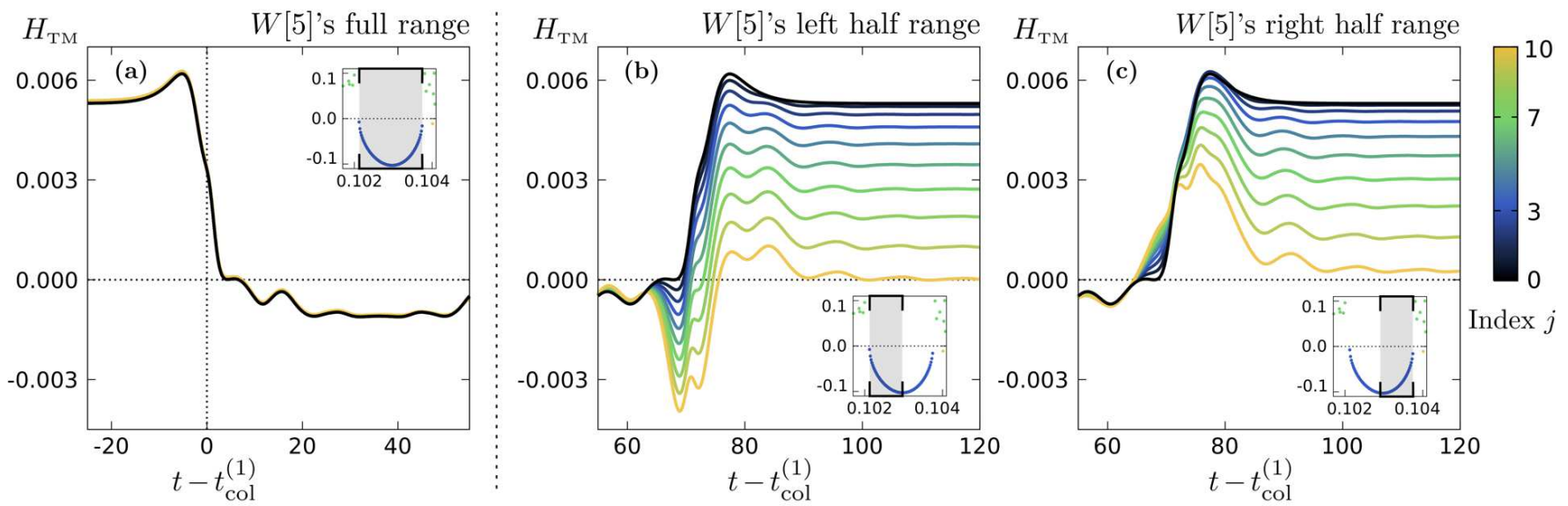

FIG. 4. (Color online) Energy of the solitons' translational mode $\left(H_{\mathrm{TM}}\right)$ as function of the time variable $t-t_{\text {col }}^{(1)}$, by considering 20 scattering processes. Here, the $v_{0}$ values for each one of these processes are taken in the $W[5]$ 's interval of regularity analogously to the plot in Fig. 3. In the panel (a), $v_{0}$ covers the full range (as indicated by the inset, containing the corresponding $v_{\infty} \times v_{0}$ plot). In this case, the 20 energy plots are almost indistinguishable. In the panels (b) and (c), $v_{0}$ covers the left and the right half range, respectively, starting from the middle point and then toward the edges (see the inset panels). In each plot, 10 processes are displayed and indexed with integers $j \in[1,10]$. The time range starts from the final point shown in panel (a). In $t-t_{\text {col }}^{(1)}=120$, the solitons will have spread out and $H_{\mathrm{TM}} \approx H_{\mathrm{TM}}^{(\infty)}$.

with all these features when the SO-coupling is present $(\gamma \neq 0)$.

In section IV, we have previously pointed out that the initial propagation velocity cannot be identified with the parameter $v_{0}$ when $\gamma \neq 0$, instead it is $v_{0}^{\prime}=v_{0}+\gamma$ as indicated by Eq. (7c) in the regime $|\xi| \gg 1$. Regarding only the effective soliton dynamics, as can be seen in heatmap plots, a pre-collisional scenario with $v_{0}=V_{0}$ and $\gamma=0$ is indistinguishable from one with $v_{0}=V_{0}-\gamma$ and $\gamma \neq 0$, since $v_{0}^{\prime}=V_{0}$ in both cases. Hence, in order to simulate the effects of the $\mathrm{SO}$-coupling over pre-collisional scenarios equivalent to those from the previous subsection, we have used a $v_{0}$ range similar to that from Fig. 2(a) translated by $\gamma$ units to the left (right) if $\gamma>0(\gamma<0)$. In Fig. 6 , the effect of the SO-coupling over the final propagation velocity $v_{\infty}^{\prime}$ is shown for several cases in which $\gamma>0$. The plots in Fig. 6(a)-(g) display similar window structures that basically differ from another one by some sort of transformation combining translation and scaling of the intervals of regularity. The critical point that separates the chaoticlike region from the regular one also translates along the $v_{0}^{\prime}$-axis as the $\gamma$ increases. One can realize that $v_{c}$ grows from Fig. 6(a) to 6(d) and diminishes from Fig. 6(d) to $6(\mathrm{~g})$. Besides these changes in the windows placement, there are new transmission windows associated with 3pass collisional scattering processes that now appear at left side of $W[2]$.

We emphasize that for $0<\gamma \lesssim 0.2$ the effect of the SO-coupling in the variational dynamics is small, in the sense that it does not affect significantly the main structure of windows and its substructures. So, the mechanism described in the previous subsection still works when the SO-coupling is present and, after some analysis of the collision dynamics within several intervals of regularity, one can verify that those interesting features associated with the reflection/transmission windows remain. We performed several iterative simulations considering $\gamma$ values gradually increasing from 0.2 up to 1.5 with step $\Delta \gamma=0.05$. By comparing the obtained plots (some of which are shown in Fig. 6(h)-(n)), one can notice that the critical velocity keeps decreasing as $\gamma$ increases, causing the whole window structure to be displaced toward the origin. Indeed, the window closest to the origin shrinks and eventually disappears when $\gamma$ reaches a certain value. The beginning of this process can be seen in the window $W[2]$ (left to right) in Fig. 6(g). As this process goes on, the structure "loses" some windows and becomes smaller. When $\gamma=0.5$ (see Fig. 6(j)), the structure can be barely seen and becomes even more confined due to the emergence of a new type of critical point that separates the chaotic-like region from a new one that extends until the origin $\left(v_{0}^{\prime}=0\right)$. This new region increases with $\gamma$ and speeds up the vanishing process of the chaotic-like region and the window structures within it, which are lastly seen in Fig. 6(1). Following, in Fig. 6(m) the window structure is gone, and only a few points can be barely seen within what is left from the chaotic-like region, which has already completely vanished in Fig. 6(m). Comparing these last two figures, we verify an inversion of the initial increasing behavior of the new region, since its interval was shortened.

Back to Fig. 6(1), we introduce a notation to differ the new type of critical velocity from the old one, with $v_{\mathcal{c}}^{\mathrm{R}}(\gamma)$ denoting the former and $v_{c}^{\mathrm{T}}(\gamma)$ the latter (previously de- 
(a)
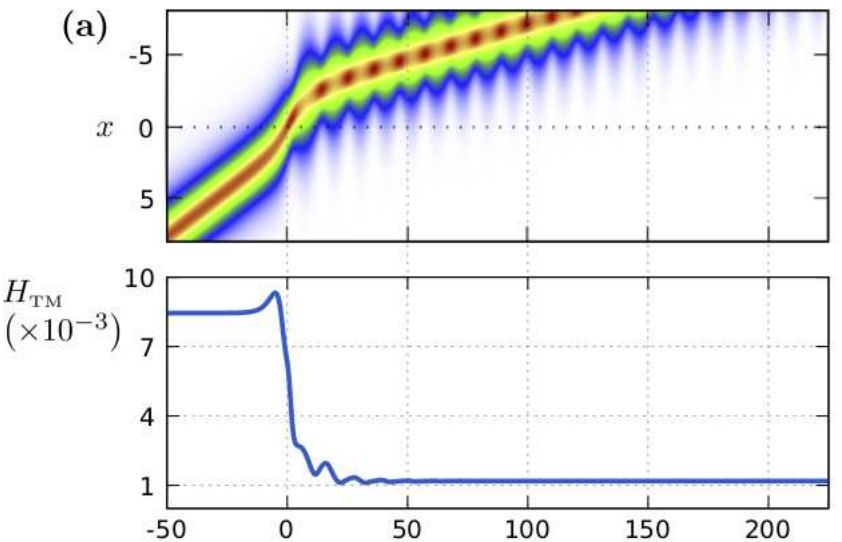

(b)
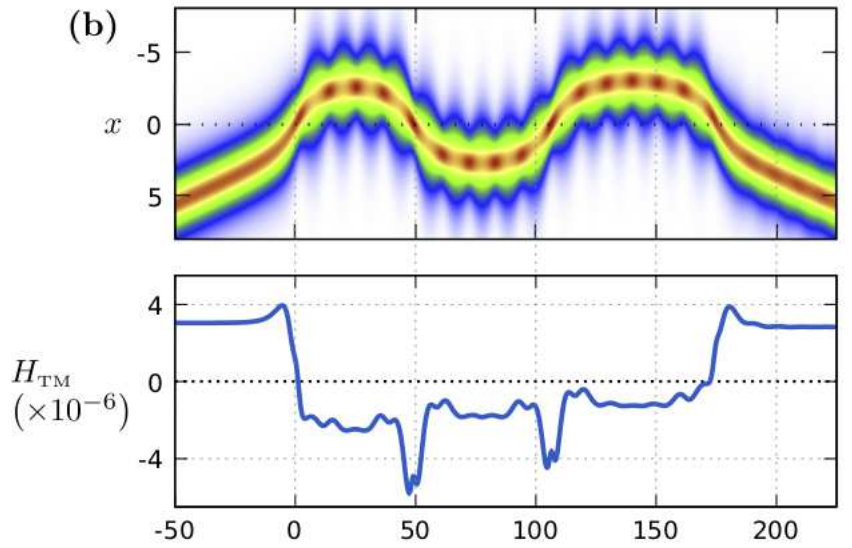

(c)

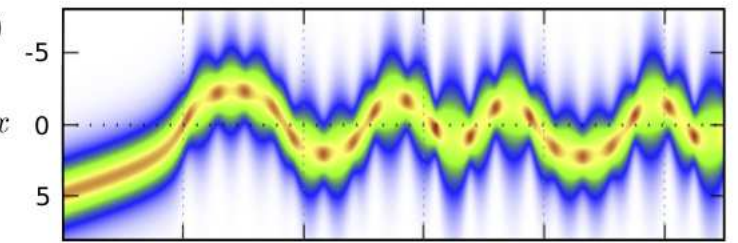

$H_{\mathrm{TM}}$

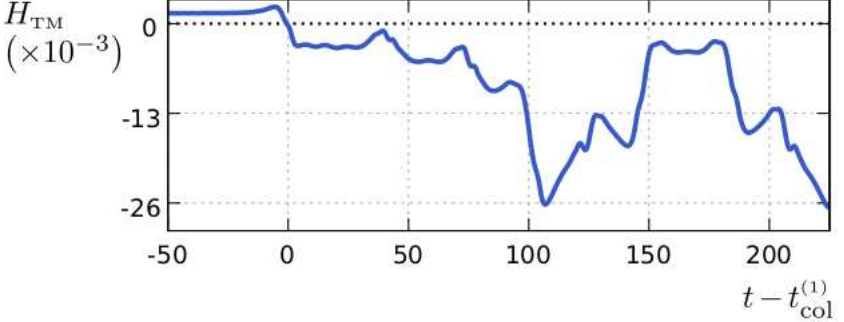

$\left|A_{-}^{\prime}\left(x, t-t_{\text {col }}^{(1)}\right)\right|$

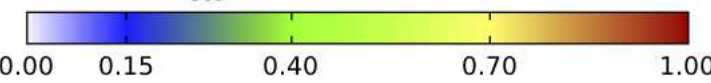

FIG. 5. (Color online) Heatmap of $\left|A_{+}^{\prime}\left(x, t-t_{\text {col }}^{(1)}\right)\right|$ and the corresponding TM energy $\left(H_{\mathrm{TM}}\right)$ versus $t-t_{\mathrm{col}}^{(1)}$, for three examples of scattering: (a) direct transmission, (b) regular scattering, and (c) irregular scattering. In panel (a) it was used $v_{0}=0.13>v_{c}$. In panel (b) it was set $v_{0}=0.077984$, belonging to $W[3,4,5]$ 's interval of regularity. In panel (c) it was considered $v_{0}=0.055$, which is located in a chaotic interval between the windows $W[2]$ and $W[3]$. noted by $v_{c}$ ). Here the dependence with $\gamma$ is written explicitly, and the superscripts $\mathrm{R}$ and $\mathrm{T}$ stand for reflection and transmission, respectively. With this notation we mean that every scattering process with $v_{0}^{\prime}>v_{c}^{\mathrm{R}}(\gamma)$ is a direct transmission, and that every scattering process with $0<v_{0}^{\prime}<v_{c}^{\mathrm{R}}(\gamma)$ is a direct reflection. The latter is a new type of regular scattering that cannot occur if $\gamma$ does not exceed a certain threshold value $\gamma_{R}$. As an example, in Fig. 6(p) it is considered two plots displaying the behavior of these two types of direct scattering process. In the direct reflection scenario (bottom plot of Fig. 6(p)) one can note that the peak position $p$ never reaches zero (without passing) and that there is no detectable shape vibrations after the collision, i.e., the scattering is practically elastic (the corresponding points in the $v_{\infty}^{\prime} \times v_{0}^{\prime}$ plots closely trace the line $y=-x \mid x \in\left[0, v_{c}^{\mathrm{R}}(\gamma)\right]$, as can be seen in Fig. 6(j)-(n)). Defining $v_{c}^{\mathrm{R}}(\gamma)=0 \forall \gamma \mid 0 \leq \gamma<\gamma_{\mathrm{R}}$, then the direct reflection critical point $\left(P_{R}\right)$ always coincides with the origin of the coordinates system (i.e., $\left.P_{R}=(0,0)\right)$, and the direct transmission one is simply $P_{T}=\left(v_{c}^{\mathrm{T}}(\gamma), 0\right)$ as usual. For $\gamma>\gamma_{R}$, the results allows one to write, in a general way, that $P_{R} \approx\left(v_{c}^{\mathrm{R}}(\gamma),-v_{c}^{\mathrm{R}}(\gamma)\right)$ and that $P_{T}=\left(v_{c}^{\mathrm{T}}(\gamma), V_{\infty}^{\mathrm{T}}(\gamma)\right)$, with the exit velocity function defined as $V_{\infty}^{\mathrm{T}}(\gamma)=f(\gamma) v_{c}^{\mathrm{T}}(\gamma)$, such that $f(\gamma)=\Theta\left(\gamma-\gamma_{R}\right) r_{\gamma}$, with $\Theta$ being the Heaviside step function and $r_{\gamma} \in[0,1]$. By graphically tracking the $P_{T}$ point, we found that $r_{\gamma}$ strictly increases with $\gamma$ and asymptotically approaches the value 1 , as shown in Fig. 6(n) where $r_{\gamma} \approx 1$, so that $P_{T}$ is very close to the line $y=x$. This means that scattering process associated with this critical point tends to become elastic one, with solitons simply crossing each other with almost no excitation of the vibrational mode.

In order to check the behavior of the critical points $P_{T}$ and $P_{R}$ with more accuracy, i.e. for a smaller $\Delta \gamma$, we developed a numerical algorithm to locate these points within a precision $\log _{10}\left(\delta v_{c}\right) \leq-5$ and without performing long iterative simulations over wide $v_{0}^{\prime}$-ranges. We set $\Delta \gamma=0.05$ and executed the algorithm for $\gamma$ values into the interval $[-2.5,2.5]$. The corresponding results are shown in Fig. 6(o). We found that the $P_{R}$ points distribution is symmetric with respect to the $\gamma=0$ axis, and also that none of these appear in the interval $\left[-\gamma_{R}, \gamma_{R}\right]$ (as indicated by our previous analysis for $\gamma>0)$. Then, we can extend the $f$ function to the negative domain by redefining it as $f(\gamma)=\Theta\left(\left|\gamma-\gamma_{\mathrm{R}}\right|\right) r_{\gamma}$, with $r_{\gamma} \approx 1$ for SO-coupling strengths $|\gamma| \gg 1$. Additionally, the length of the direct reflection region is maximum, $\max \left[v_{c}^{\mathrm{R}}(\gamma)\right]$, when $|\gamma|$ is about 1.15 , and, $v_{c}^{\mathrm{R}}(|\gamma|)$ strictly decreases for greater SO-coupling strengths. Regarding the $P_{T}$ points distribution, we observe that it is not symmetric and displays a special behavior when $\gamma \in\left[0, \gamma_{\mathrm{R}}\right]$. In this interval, one notes that, for a certain SO-coupling strength $\gamma_{\mathrm{T}}>0$, the length of the chaoticlike region is maximum (i.e., $\max \left[v_{c}^{\mathrm{T}}(\gamma)\right]=v_{c}^{\mathrm{T}}\left(\gamma_{\mathrm{T}}\right)$ ). By reducing the discretization to $\Delta \gamma=0.0125$ over the interval $[0,0.25]$ (highlighted by an arrow in Fig. $6(0)$ ), we 

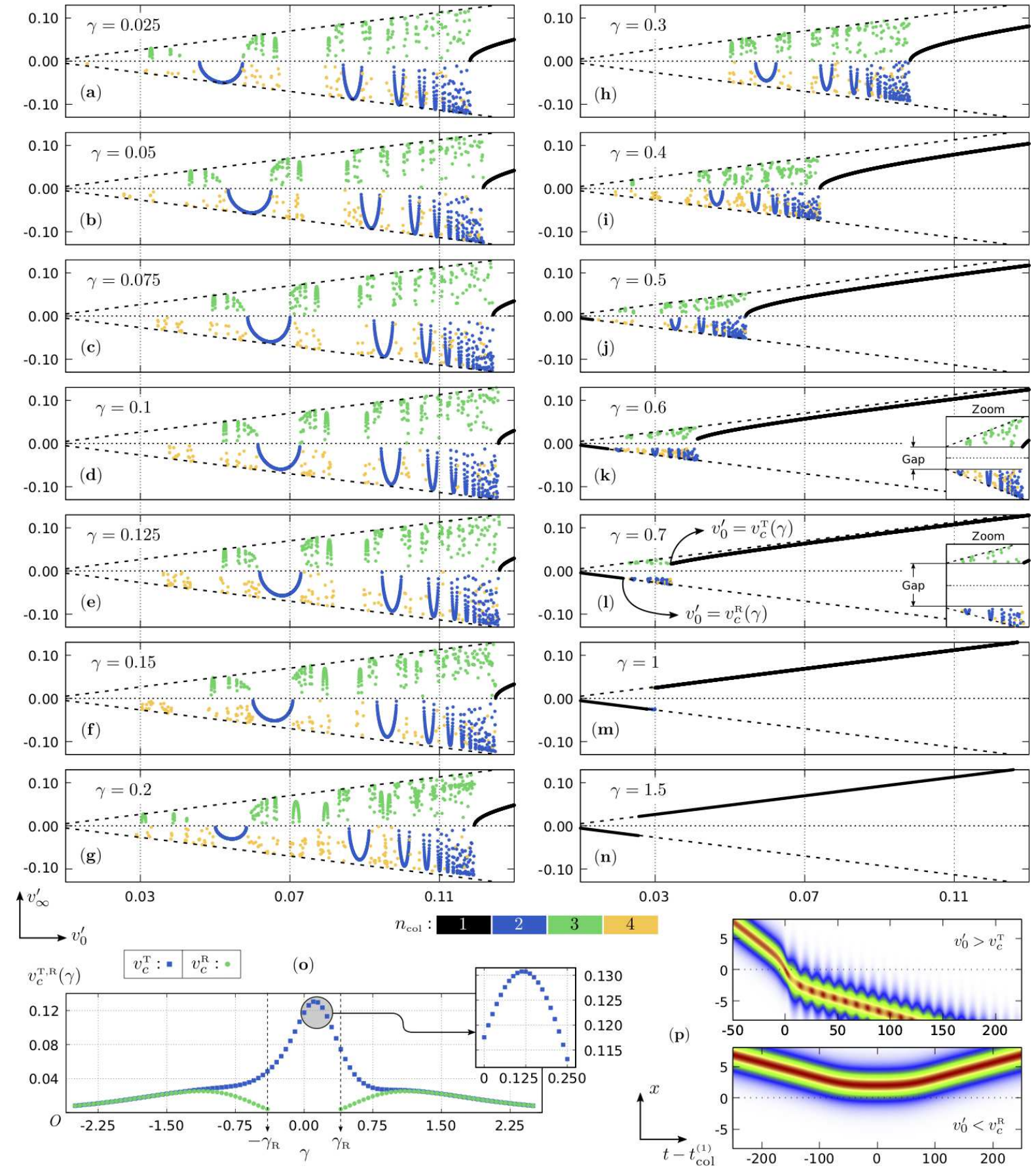

FIG. 6. (Color online) Scattering results for $v_{\infty}^{\prime}$ versus $v_{0}^{\prime}$ obtained via iterative simulations of the reduced ODE model (Eqs. (7a)$(7 \mathrm{~d})$ ) in a fixed $v_{0}{ }^{\prime}$-range for different values of $\gamma>0$, starting from $\gamma=0.025$ in panel (a) and adding $\Delta \gamma=0.025$ at each step until $\gamma=0.15$ in panel (f). Next, we start from $\gamma=0.2$ in panel (g) and adding $\Delta \gamma=0.1$ at each step until $\gamma=0.7$ in panel (1). The SO-coupling parameter is chosen as $\gamma=1.0$ and $\gamma=1.5$ in panels $(\mathrm{m})$ and (n), respectively. At the right corner of the plots in (k) and (l), a zoom of the window structure is displayed to highlight the emerging gap that splits the chaotic-like region in two parts. In panel (o), the approximate values for the two types of critical velocity, $v_{c}^{\mathrm{R}}(\gamma)$ and $v_{c}^{\mathrm{T}}(\gamma)$, are shown in two graphs, with the smallest one focusing in the $\gamma$-range where $v_{c}^{\mathrm{T}}(\gamma)$ reaches a peak value. In panel $(\mathrm{p})$, two heatmap plots are displayed to exemplify the characteristic dynamics of the two types of direct scattering, namely, direct reflection $\left(v_{0}^{\prime} \leq v_{c}^{\mathrm{R}}(\gamma)\right)$ and direct transmission $\left(v_{0}^{\prime} \geq v_{c}^{\mathrm{T}}(\gamma)\right)$.

obtained that $\gamma_{\mathrm{T}}$ is about 0.1125 . Indeed, this result was expected since such behavior could be inferred from our previous analysis for $\gamma>0$. The asymmetric behavior of $v_{c}^{\mathrm{T}}(\gamma)$ displayed in Fig. 6(o) is explained as follows. 
For a SO-coupling strength $\left|\gamma^{\prime}\right|$, there are always two initial phases giving the same initial propagation velocity $V_{0}$, which are $v_{0}^{ \pm}=V_{0} \pm\left|\gamma^{\prime}\right|$ for $\gamma=\mp\left|\gamma^{\prime}\right|$. The first term in Eq. (7c) is simply $-v^{\prime}$, hence it is equal to $-V_{0}$ for both initial conditions $v_{0}^{ \pm}$. Now, if the dependence of the coupling function $G$ with variational parameter $v$ was through a term proportional to $v^{\prime}$, then the reduced model would be clearly symmetric with respect to $\gamma$. However, this is not the case here, because the Rabi coupling has broken the SO-coupling inversion symmetry.

Regarding the rest of the $P_{T}$ distribution points residing in the intervals $\left[-2.5,-\gamma_{\mathrm{R}}\right]$ and $\left[\gamma_{\mathrm{R}}, 2.5\right]$, the data shows that $v_{c}^{\mathrm{T}}(\gamma)$ strictly decreases for increasing $|\gamma|$. Also, from Fig. 6(o), we observe that when $|\gamma| \gtrsim \max \left[v_{c}^{\mathrm{R}}\right]$ the difference given by $v_{c}^{\mathrm{T}}(|\gamma|)-v_{c}^{\mathrm{R}}(|\gamma|)$ (length of the chaotic-like region) is of the order of $10^{-4}$ and quickly approaches $0^{+}$as $|\gamma|$ grows, i.e., the $P_{T}$ and $P_{R}$ points tend to coalesce for large values of the SOcoupling strength. In the regime $|\gamma| \gg 1$, one can infer from the behavior of the critical points that $v_{c}^{\mathrm{TR}}(|\gamma|) \approx 0$, therefore the scattering tends to become a simple elastic direct transmission for any pre-collisional scenario $\left(\forall v_{0}^{\prime}>0\right)$, which is equivalent to turning off the Rabi coupling.

By following the same protocol employed in the previous subsection, we considered here the cases in which $\gamma= \pm 0.15$ and investigated some substructures. As example, in Figs. 7)(a)-(c) we display the case with $\gamma=0.15$ (similar results are found for the case with negative sign). When analyzing the window distributions, we found that the pattern associated with reflection windows differs from the one associated with transmission windows, with the former having an overall larger window spacing compared with the latter. However, for the case $\gamma=-0.15$ one finds an opposite behavior. Hence, the results indicate that the fractal-like behavior can indeed persist if the first window structure is weakly affected by the SO-coupling, and that the changes in the window patterns depend of the sign of $\gamma$. We also explored some cases in which the SO-coupling strength caused the chaotic-like regions to become very small as in Figs. 6(j)-(k). So, we found that the first substructures still emerge in the edges of the remaining windows that were not significantly affected by the vanishing process previously described.

Hitherto, we have focused on the emergent effects caused by the SO-coupling, hence our analyses considered only the general aspects regarding the two types of regular scattering and their associated intervals, with more emphasis in the intertwining chaotic-like interval and window structures within it. We have firstly investigated how the parameter $\gamma$ modifies the coupling function $G$ and its derivatives $\partial_{q} G$. To this end, we rewrite the Eq. (5) in terms of the propagation velocity by making $v=v^{\prime}-\gamma$, which is equivalent to the variable exchange $\zeta \rightarrow \zeta^{\prime}-2 \gamma$, with $\zeta^{\prime}=2 v^{\prime}+\xi b$ being analogously to $\zeta$ in the case of $\gamma=0$. Then, defining $G^{\prime}$ given
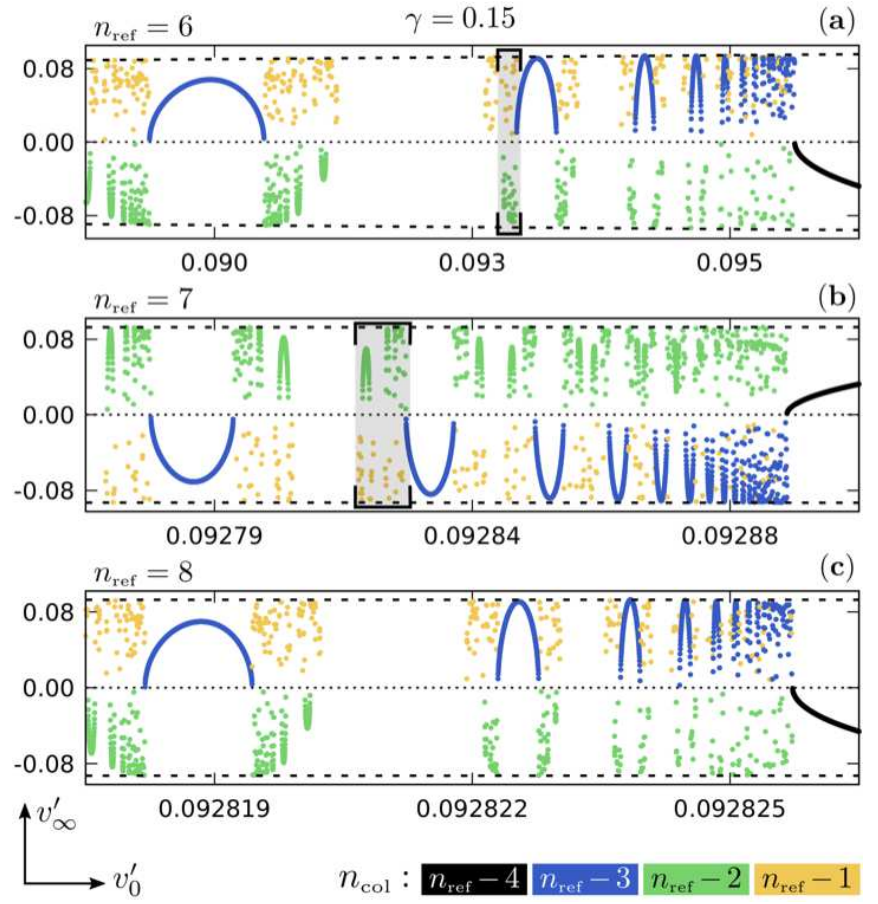

FIG. 7. (Color online) Scattering results for $v_{\infty}^{\prime}$ versus $v_{0}^{\prime}$ obtained via iterative simulations of the reduced ODE model (Eqs. (7a)-(7d)) in three $v_{0}{ }^{\prime}$-ranges (a)-(c) within the interval $\left(0, v_{c}^{\mathrm{T}}(\gamma)\right]$ with $\gamma=0.15$. The two highlighted regions (gray) indicate the $v_{0}{ }^{\prime}$-range of the plot immediately below. The panel (a) corresponds to a "zoom" of the $v_{0}^{\prime}$-range containing a substructure near the left edge of the second window (from left to right) of the main structure displayed in Fig. 6(f).

by

$$
\begin{aligned}
G^{\prime}\left(\xi, \zeta^{\prime}, w\right) & =\frac{\sin \left(\zeta^{\prime} p-2 \gamma p\right)}{\sinh (\xi) \sinh \left(\pi \zeta^{\prime} w / 2-\pi \gamma w\right)}, \\
\left.G^{\prime}\right|_{b=0, w=1} & =\frac{\sin \left[2 p\left(v^{\prime}-\gamma\right)\right]}{\sinh (2 p) \sinh \left[\pi\left(v^{\prime}-\gamma\right)\right]} \quad(|\xi| \gg 1),
\end{aligned}
$$

with the Eq. (18) valid before the collision. We stress that in the case of $\gamma=0$, the Eq. (17) recovers the form of $G$ obtained in the previous section, i.e., $\left[G^{\prime}, \zeta^{\prime}, v^{\prime}\right]_{\gamma=0}=[G, \zeta, v]$ (see Eq. (5)). We performed an extensive study of the above functions to figure out how the terms $2 \gamma p$ and $\pi \gamma w$ modify the variational dynamics, with focus on the derivatives $\partial_{p} G^{\prime}$ and $\partial_{v} G^{\prime}$, which are associated with the translational acceleration terms in the reduced model and develop a more important role in the propagation dynamics. By this study we retrieved the most important qualitative aspects of the SO-coupling influence over the interaction.

Considering only the denominator of Eq. (17), the term $\pi \gamma w$ alters the interaction strength in different ways depending on the behavior of the width parameter $w$. During the bound-states, the oscillatory character of $w$ due shape vibrations induces oscillations in 
the Rabi interaction strength, which are small when $\pi|\gamma| w \ll 1$, i.e., if $|\gamma| \ll 1$. For greater SO-coupling strengths, this oscillation can make the bound-state dynamics very complicated, as the binding strength keeping the solitons together alternates between weak and strong regimes. When the SO-coupling is such that $|\gamma| \gg v^{\prime}$, the leading effect of the term $\pi \gamma w$ is the dumping of the Rabi interaction strength, as one can clearly verify from Eq. (18). This can be related to the behavior of the critical point $P_{T}$, because, as the Rabi interaction weakens due to the increasing $|\gamma|$, the maximum propagation velocity for the bound-state formation $\left(v_{c}^{\mathrm{T}}(\gamma)\right.$ ) reduces until a certain value in which the attraction is still enough to trap the solitons. On the other hand, regarding now the numerators of Eqs. (17) and (18), one notes that the parameter $\gamma$ induces oscillations that develop when the solitons are moving, which occur at a fixed frequency $2|\gamma|$ when $p$ varies linearly during precollisional scenarios. This leads to oscillations in the sign of every term containing a derivative of $G^{\prime}$, causing the Rabi interaction to oscillate between regimes of attraction $\left(\Gamma \partial_{p, v} G^{\prime}<0\right)$ and repulsion $\left(\Gamma \partial_{p, v} G^{\prime}>0\right)$. Since the denominator of Eq. (18) is dominated by the term $\sinh (2 p) \gg 1$, the approximation $G^{\prime}, \partial_{q} G^{\prime} \approx 0$ is valid and the sign of the coupling terms do not matter during pre-collisional scenarios. Therefore, the sign oscillation become relevant only when $p$ is small enough so that the translational acceleration terms, $\Gamma \partial_{p, v} G^{\prime}$, can significantly alter the propagation. During the bound-states, $p$ is confined to a narrow interval of values $(|p| \lesssim 5)$, if the SO-coupling strength is small, such that $2|\gamma p| \ll 1$, then the sign oscillation barely alters the predominantly attractive Rabi interaction. In contrast, for greater SOcoupling strengths, $2\left|\gamma_{p}\right|$ is not small and such oscillations are much more prominent, making the oscillations of the bound-state to vary in an unpredictable way. For instance, one of the consequences of this non trivial behavior is displayed in Figs. 6(k)-(1), where one can see a gap in the chaotic-like region that splits it into two smaller regions, i.e., there is a forbidden range of final velocities that establishes a threshold value for $\left|v_{\infty}^{\prime}\right|$ if $v_{0}^{\prime} \in\left[v_{c}^{\mathrm{R}}(\gamma), v_{c}^{\mathrm{T}}(\gamma)\right]$. This effect happens because the Rabi interaction becomes momentarily repulsive just after the unbinding, and then, due the proximity of the solitons, the acceleration is greater enough to increase the propagation velocity. The gain in velocity is greater as greater the SO-coupling strengths is and also when the acceleration acts for a longer time, i.e., if $v^{\prime}$ is very small just after the unbinding (as in those regular inelastic processes near the window edges). This increasing gap explains the behavior of the parameter $r_{\gamma}$ in the $P_{T}$ critical point expression, since $V_{\infty}^{\mathrm{T}}(\gamma)$ follows the gap upper boundary.

The alternation between attractive and repulsive Rabi interaction can be directly related with the emergence of the direct reflection region. We investigated several scattering processes with $v_{0}^{\prime} \in\left(0, v_{c}^{\mathrm{R}}(\gamma)\right]$ for various values of $\gamma$, observing that the role of the variational parame- ters $w$ and $b$ is negligible. Indeed, this occurs because the collision is quasi-elastic, with the energy stored within the vibrational mode being practically zero when compared with the energy within the translational mode. This finding allowed us to study this type of scattering in a more quantitative way, since we can set $w=1$ and $b=0$ to obtain the effective reduced ODE model

$$
\dot{v}=\pi \Gamma \frac{\partial G_{0}^{\prime}}{\partial p} \quad, \quad \dot{p}=-\left(v^{\prime}+\pi \Gamma \frac{\partial G_{0}^{\prime}}{\partial v}\right),
$$

with $G_{0}^{\prime}=\left.G^{\prime}\right|_{b=0, w=1}$ being the effective coupling function yielded by Eq. (18). Note that if $\gamma=0, G_{0}^{\prime}$ coincides with $G_{0}$ of Eq. (9), introduced in section IV. We study the phase space trajectories governed by Eq. (19) subjected to the initial conditions $\left(p_{0}, v_{0}^{\prime}\right)$, with $p_{0}=10$ as usual and $v_{0}^{\prime}(i)=V_{0}^{(\text {init })}+\Delta_{\gamma}(i-1) \mid i \in[1,2, \ldots, 8]$, with $\Delta_{\gamma}=\left(v_{c}^{\mathrm{R}}(\gamma)-V_{0}^{\text {(init) }}\right) /(I-1)$ and $V_{0}^{\text {(init) }}=0.001$. When these phase space trajectories (two-dimensional curves) are plotted with the contour line plot of $\partial_{p} G_{0}^{\prime}$ or $\partial_{v} G_{0}^{\prime}$ in the background, one can visualize how the propagation is driven by the oscillatory Rabi interaction, and also how the SO-coupling strength increases the frequency of such oscillations and consequently alters the dynamics. This is exactly what is displayed in Fig. 8 for three different values of $\gamma>0$ and with background composed by the contour line plots of $\partial_{p} G_{0}^{\prime}$. The corresponding negative values provide the same results and similar plots are obtained when $\partial_{v} G_{0}^{\prime}$ is considered instead. The alternation between attraction (gray zones with $\Gamma \partial_{p} G_{0}^{\prime}>0$ ) and repulsion (white zones with $\Gamma \partial_{p} G_{0}^{\prime}<0$ ) is clearly depicted in Fig. 8. Considering the case with $\gamma=0.5$, the trajectories show that the attraction zone immediately affecting all the processes in the far field (close to $p=10)$ is negligible $\left(\partial_{p, v} G_{0}^{\prime} \approx 0\right)$ due to the initially large separation. As $p$ reduces and reaches the repulsion zone $(p \simeq 6)$, the separation becomes small enough to cause a deceleration that can act during a long enough time interval to completely break the solitons $\left(v^{\prime}=0\right)$, and then accelerate them away $\left(v^{\prime}<0\right)$ back to the far field in such way that, in the post-collisional scenario, $v_{\infty}^{\prime} \approx-v_{0}^{\prime}$. Also, one can see that the shortest trajectory $\left(v_{0}^{\prime}=0.001\right)$ quickly turns back as it gets into the repulsion zone, and that the longest trajectory $\left(v_{0}^{\prime} \approx v_{c}^{\mathrm{R}}(\gamma)\right)$ turns back after almost reaching the attraction zone that extends all the way toward $p=0$.

Regarding the other two cases, with $\gamma=1.0$ and $\gamma=1.5$, an analogous behavior can be visualized. However, due to the greater SO-coupling strengths, there is more zones of attraction and repulsion that add more details to the dynamics. In both cases, the effect of the attraction/repulsion zones in the far field are once again negligible, and most of trajectories begin to be significantly affected after reaching the next-to-last repulsion zone, which is the zone where the shortest trajectory turns back before reaching the last and most effective repulsion one (see Fig. 8). In the case with $\gamma=1.0$, one observes that the next-to-last repulsion zone barely in- 

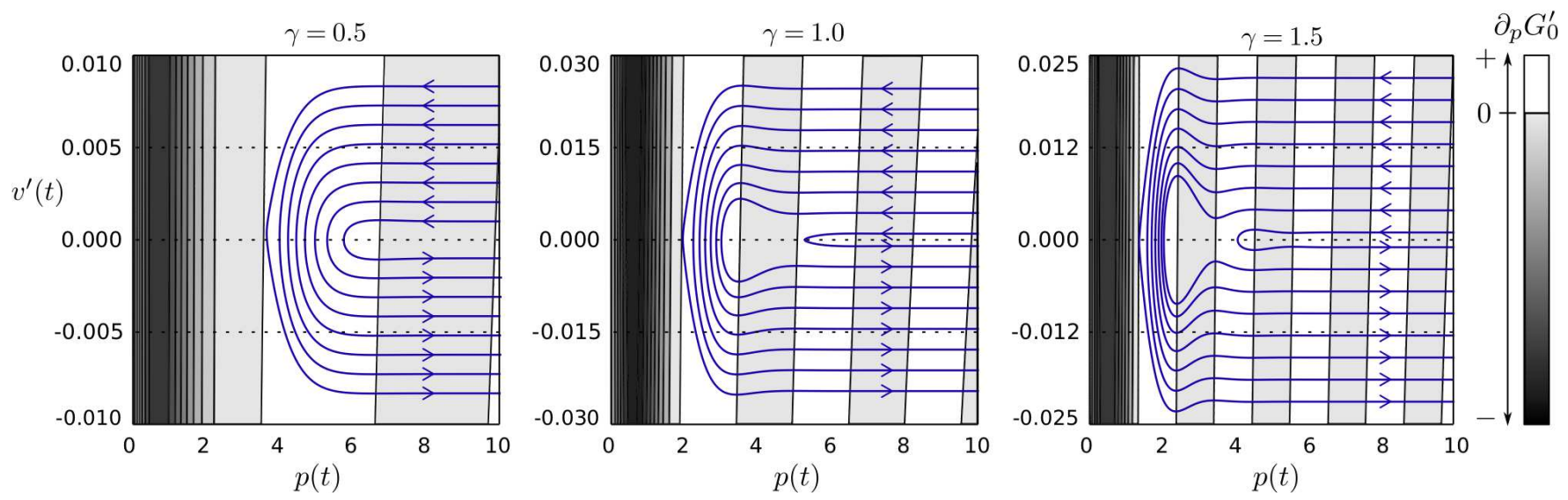

FIG. 8. (Color online) Phase space trajectories governed by the effective reduced ODE model (given by Eqs. (19)) providing a variational description for the direct reflection type of solitons scattering. In the three cases considered $(\gamma=0.5,1.0,1.5)$, a total of 8 trajectories with $p_{0}=10$ and $v_{0}^{\prime} \in\left(0, v_{c}^{\mathrm{R}}(\gamma)\right]$ are plotted. The background is a contour line plot of the function $\partial_{p} G_{0}^{\prime}$. Since $\Gamma=-0.04<0$, the attraction zones are the highlighted by gray/black regions, corresponding to sgn $\left(\partial_{p} G_{0}^{\prime}\right)=-1$, while repulsion zones are identified by the white regions, corresponding to $\operatorname{sgn}\left(\partial_{p} G_{0}^{\prime}\right)=+1$.

fluences the other trajectories $\left(v_{0}^{\prime}>0.001\right)$, which make the way through the attraction zone until finally reaching the last repulsion one and then turning back. In addition, in the case with $\gamma=1.5$, these final zones are narrower and closer to the $p=0$ axis, hence the acceleration effects are amplified causing the trajectories to assume the shapes as seen in Fig. 8. For greater SO-coupling strengths, the zones depicted in this figure keep getting narrower and closer to the $p=0$ axis. The effectiveness of the acceleration and deceleration under the trajectories diminishes and the maximum velocity for the occurrence of direct reflection scattering becomes smaller (this connects with the decreasing behavior of $v_{c}^{R}(|\gamma|)$ for $|\gamma| \gtrsim 1.15)$. As $|\gamma|$ increases further, the effects of the repulsion and attraction zones cancel each other out (in average). Also, in this case the Rabi interaction is weakened, i.e., the scattering tends to be a mere direct transmission for almost all $v_{0}^{\prime}>0$.

\section{CONCLUSION}

In summary, we investigated the influence of the SO coupling on the collisional dynamics of solitons in binary BECs by using a reduced ordinary differential equations (ODE) model based on a variational approach, which allow us to analytically investigate the formation of fractal-like patterns and the properties of the scattered solitons. To this end, we first studied the collision of solitons in the absence of SO coupling and then we started to verify the influence on the scattering patterns by changing the value of the $\mathrm{SO}$ coupling parameter $\gamma$. We found exotic structures of scattering by focusing on the values of the exit velocities $v_{\infty}^{\prime}$ for given input velocities $v_{0}^{\prime}$. Also, we verified that these structures present a fractal-like pattern, i.e., periodic repetitions of the main structure in its substructures, corresponding to the zoomed views. The size of the region presenting windows structures is drastically affected by the SO coupling. Indeed, we observe that for $|\gamma| \gtrsim 1.15$ the structure of windows vanishes completely. Also, the SO-coupling promotes non-trivial oscillations in the Rabi interaction strength and its sign, which are the sources of the emergent effects altering the window structure that vanishes as the chaotic-like region is compressed in the $v_{0}^{\prime}$-direction by the regions of direct transmission and direct reflection, and in the $v_{\infty}^{\prime}$ direction by the growing gap of forbidden final propagation velocities.

\section{Acknowledgments}

We acknowledge financial support from the Brazilian agencies CNPq, CAPES, FAPEG, and the National Institute of Science and Technology (INCT) for Quantum Information.
[1] Y.-J. Lin, K. Jiménez-García, and I. B. Spielman, Nature 471, 83 (2011).

[2] M. Merkl, A. Jacob, F. E. Zimmer, P. Öhberg, and L. Santos, Phys. Rev. Lett. 104, 073603 (2010).
[3] X.-Q. $\mathrm{Xu}$ and J. H. Han, Phys. Rev. Lett. 107, 200401 (2011).

[4] J. Radić, T. A. Sedrakyan, I. B. Spielman, and V. Galitski, Phys. Rev. A 84, 063604 (2011). 
[5] B. Ramachandhran, B. Opanchuk, X.-J. Liu, H. Pu, P. D. Drummond, and H. Hu, Phys. Rev. A 85, 023606 (2012).

[6] V. E. Lobanov, Y. V. Kartashov, and V. V. Konotop, Phys. Rev. Lett. 112, 180403 (2014).

[7] H. Sakaguchi, B. Li, and B. A. Malomed, Phys. Rev. E 89, 032920 (2014).

[8] T. Kawakami, T. Mizushima, M. Nitta, and K. Machida, Phys. Rev. Lett. 109, 015301 (2012).

[9] G. J. Conduit, Phys. Rev. A 86, 021605 (2012).

[10] O. Fialko, J. Brand, and U. Zülicke, Phys. Rev. A 85, 051605 (2012).

[11] V. Achilleos, J. Stockhofe, P. G. Kevrekidis, D. J. Frantzeskakis, and P. Schmelcher, EPL (Europhysics Lett. 103, 20002 (2013).

[12] V. Achilleos, D. J. Frantzeskakis, P. G. Kevrekidis, and D. E. Pelinovsky, Phys. Rev. Lett. 110, 264101 (2013).

[13] Y. V. Kartashov, V. V. Konotop, and F. K. Abdullaev, Phys. Rev. Lett. 111, 060402 (2013).

[14] Y. Zhang, Y. Xu, and T. Busch, Phys. Rev. A 91, 043629 (2015).

[15] H. Sakaguchi and B. A. Malomed, Phys. Rev. A 97, 013607 (2018).

[16] D. B. Belobo and T. Meier, Sci. Rep. 8, 3706 (2018).

[17] T.-L. Ho and S. Zhang, Phys. Rev. Lett. 107, 150403 (2011).

[18] S. Sinha, R. Nath, and L. Santos, Phys. Rev. Lett. 107, 270401 (2011).

[19] D.-W. Zhang, L.-B. Fu, Z. D. Wang, and S.-L. Zhu, Phys. Rev. A 85, 043609 (2012).

[20] M. A. Garcia-March, G. Mazzarella, L. Dell'Anna, B. Juliá-Díaz, L. Salasnich, and A. Polls, Phys. Rev. A 89, 063607 (2014).

[21] W.-Y. Wang, J. Liu, and L.-B. Fu, Phys. Rev. A 92, 053608 (2015).

[22] R. A. Williams, L. J. LeBlanc, K. Jimenez-Garcia, M. C. Beeler, A. R. Perry, W. D. Phillips, and I. B. Spielman, Science (80-. ). 335, 314 (2012).

[23] L. J. LeBlanc, M. C. Beeler, K. Jiménez-García, A. R. Perry, S. Sugawa, R. A. Williams, and I. B. Spielman, New J. Phys. 15, 073011 (2013).

[24] C. Qu, C. Hamner, M. Gong, C. Zhang, and P. Engels, Phys. Rev. A 88, 021604 (2013)

[25] V. Achilleos, D. J. Frantzeskakis, and P. G. Kevrekidis, Phys. Rev. A 89, 033636 (2014).

[26] K. Jiménez-García, L. J. LeBlanc, R. A. Williams, M. C. Beeler, C. Qu, M. Gong, C. Zhang, and I. B. Spielman, Phys. Rev. Lett. 114, 125301 (2015).

[27] P. Zou, J. Brand, X.-J. Liu, and H. Hu, Phys. Rev. Lett. 117, 225302 (2016).

[28] Y. V. Kartashov and V. V. Konotop, Phys. Rev. Lett. 118, 190401 (2017).

[29] M. A. Khamehchi, K. Hossain, M. E. Mossman, Y. Zhang, T. Busch, M. M. Forbes, and P. Engels, Phys. Rev. Lett. 118, 155301 (2017).

[30] L. Salasnich and B. A. Malomed, Phys. Rev. A 87, 063625 (2013).

[31] Y. $\mathrm{Xu}, \mathrm{Y}$. Zhang, and B. Wu, Phys. Rev. A 87, 013614 (2013).

[32] D. A. Zezyulin, R. Driben, V. V. Konotop, and B. A. Malomed, Phys. Rev. A 88, 013607 (2013).

[33] Y. V. Kartashov, V. V. Konotop, and D. A. Zezyulin, Phys. Rev. A 90, 063621 (2014).

[34] H. Sakaguchi and B. A. Malomed, Phys. Rev. E 90, 062922 (2014).

[35] E. Chiquillo, Laser Phys. 24, 085502 (2014).
[36] S. Gautam and S. K. Adhikari, Phys. Rev. A 91, 013624 (2015).

[37] S. Cao, C.-J. Shan, D.-W. Zhang, X. Qin, and J. Xu, J. Opt. Soc. Am. B 32, 201 (2015).

[38] L. Wen, Q. Sun, Y. Chen, D.-S. Wang, J. Hu, H. Chen, W.-M. Liu, G. Juzeliunas, B. A. Malomed, and A.-C. Ji, Phys. Rev. A 94, 061602 (2016).

[39] Y.-E. Li and J.-K. Xue, Chinese Phys. Lett. 33, 100502 (2016).

[40] H. Sakaguchi and B. A. Malomed, Phys. Rev. A 96, 043620 (2017).

[41] Y. Li, Z. Luo, Y. Liu, Z. Chen, C. Huang, S. Fu, H. Tan, and B. A. Malomed, New J. Phys. 19, 113043 (2017).

[42] L. Salasnich, W. B. Cardoso, and B. A. Malomed, Phys. Rev. A 90, 033629 (2014)

[43] B. Liao, S. Li, C. Huang, Z. Luo, W. Pang, H. Tan, B. A. Malomed, and Y. Li, Phys. Rev. A 96, 043613 (2017).

[44] Y. Li, Y. Liu, Z. Fan, W. Pang, S. Fu, and B. A. Malomed, Phys. Rev. A 95, 063613 (2017).

[45] M. Kato, X.-F. Zhang, and H. Saito, Phys. Rev. A 95, 043605 (2017).

[46] C. Huang, Y. Ye, S. Liu, H. He, W. Pang, B. A. Malomed, and Y. Li, Phys. Rev. A 97, 013636 (2018).

[47] Y.-K. Liu and S.-J. Yang, EPL (Europhysics Lett. 108, 30004 (2014).

[48] R. M. Wilson, B. M. Anderson, and C. W. Clark, Phys. Rev. Lett. 111, 185303 (2013).

[49] X. Jiang, Z. Fan, Z. Chen, W. Pang, Y. Li, and B. A. Malomed, Phys. Rev. A 93, 023633 (2016).

[50] S. Gautam and S. K. Adhikari, Phys. Rev. A 95, 013608 (2017).

[51] G. Chen, Y. Liu, and H. Wang, Commun. Nonlinear Sci. Numer. Simul. 48, 318 (2017).

[52] Y.-C. Zhang, Z.-W. Zhou, B. A. Malomed, and H. Pu, Phys. Rev. Lett. 115, 253902 (2015).

[53] J. Yang and Y. Tan, Phys. Rev. Lett. 85, 3624 (2000).

[54] Y. Tan and J. Yang, Phys. Rev. E 64, 056616 (2001).

[55] S. V. Dmitriev and T. Shigenari, Chaos An Interdiscip. J. Nonlinear Sci. 12, 324 (2002).

[56] S. V. Dmitriev, D. A. Semagin, A. A. Sukhorukov, and T. Shigenari, Phys. Rev. E 66, 046609 (2002).

[57] Y. Zhu and J. Yang, Phys. Rev. E 75, 036605 (2007).

[58] Y. Zhu, R. Haberman, and J. Yang, Phys. Rev. Lett. 100, 143901 (2008).

[59] Y. Zhu, R. Haberman, and J. Yang, Phys. D Nonlinear Phenom. 237, 2411 (2008).

[60] Y. Zhu, R. Haberman, and J. Yang, Stud. Appl. Math. 122, 449 (2009).

[61] A. Hause, H. Hartwig, and F. Mitschke, Phys. Rev. A 82, 053833 (2010).

[62] R. M. Teixeira and W. B. Cardoso, Phys. Lett. A 380, 2738 (2016).

[63] R. H. Goodman, Chaos An Interdiscip. J. Nonlinear Sci. 18, 023113

[64] R. H. Goodman, A. Rahman, M. J. Bellanich, and C. N. Morrison, Chaos An Interdiscip. J. Nonlinear Sci. 25, 043109 (2015).

[65] K. Fukushima and T. Yamada, Phys. Lett. A 200, 350 (1995).

[66] M. Higuchi, K. Fukushima, and T. Yamada, Chaos, Solitons and Fractals 9, 845 (1998).

[67] S. V. Dmitriev, Y. S. Kivshar, and T. Shigenari, Phys. Rev. E 64, 056613 (2001).

[68] S. V. Dmitriev, Y. S. Kivshar, and T. Shigenari, Phys. B Condens. Matter 316-317, 139 (2002). 
[69] S. V. Dmitriev, P. G. Kevrekidis, and Y. S. Kivshar, Phys. Rev. E 78, 046604 (2008).

[70] C. Hamner, Y. Zhang, M. A. Khamehchi, M. J. Davis, and P. Engels, Phys. Rev. Lett. 114, 070401 (2015).

[71] S. Inouye, M. R. Andrews, J. Stenger, H.-J. Miesner, D. M. Stamper-Kurn, and W. Ketterle, Nature 392, 151 (1998).
[72] J. Yang, Nonlinear Waves in Integrable and Nonintegrable Systems (Society for Industrial and Applied Mathematics, 2010). 